\title{
Size-dependent properties of simulated 2-D solar granulation
}

\author{
A.S. Gadun ${ }^{1, \dagger}$, A. Hanslmeier ${ }^{4}$, K.N. Pikalov ${ }^{1}$, S.R.O. Ploner ${ }^{2,3}$, K.G. Puschmann ${ }^{4}$, and S.K. Solanki ${ }^{2,3}$ \\ 1 Main Astronomical Observatory, Goloseevo, 03680 Kiev-127, Ukraine \\ 2 Max Planck Institute for Aeronomy, D-37191 Katlenburg-Lindau, Germany \\ 3 Institut für Astronomie, ETH-Zentrum, CH-8092 Zürich, Switzerland \\ ${ }^{4}$ Institute for Geophysics, Astrophysics and Meteorology, Universitätsplatz 5, A-8010 Graz, Austria
}

Received April 14; accepted July 26, 2000

\begin{abstract}
Two time-dependent sets of two-dimensional hydrodynamic models of solar granulation have been analyzed to obtain dependence of simulated thermal convection on the horizontal size of the convection cells. The two sets of models treat thermal convection either as fully non-stationary, multiscale convection (granular convection is a surface phenomenon) or as quasi-steadystate convection cells (they treat granular convection as a collection of deep-formed cells). The following results were obtained:
\end{abstract}

1) quasi-steady convection cells can be divided into 3 groups according to their properties and evolution, namely small-scale (up to $L \sim 900 \mathrm{~km}$ ), intermediatescale $(1000-1500 \mathrm{~km})$ and large-scale (larger $1500 \mathrm{~km}$ ) convection cells. For the first group thermal damping due to radiative exchange of energy, mostly in the horizontal direction, is very important. Large-scale cells build up a pressure excess, which can lead to their total fragmentation. Similar processes also acts on the fully non-stationary convection.

2) The largest horizontal size of convection cells for which steady-state solutions can be obtained is about $1500 \mathrm{~km}$. This corresponds to granules, i.e. the bright parts of the convection cells, with a diameter of about $1000 \mathrm{~km}$.

3) In addition to the zone of high convective instability associated with the partial ionization of hydrogen, we identify another layer harboring important dynamic processes in steady-state models. Just below the hydrogen-ionization layer pressure fluctuations and the acoustic flux are reduced. Steady-state models with reflecting lateral boundaries even exhibit an inversion of pressure fluctuations there.

4) From observational point of view the surface convection differs from steady-state deep treatment of thermal convection in the dependence of vertical granular velocities

Send offprint requests to: A. Hanslmeier, e-mail: arnold.hanslmeier@kfunigraz.ac.at

$\dagger$ deceased. on their sizes for small-scale inhomogeneous. However, they cannot be distinguished by the dependence of temperature or emergent intensity of brightness structures.

5) Both kinds of models demonstrate the inversion of density in subphotospheric layers. It is more pronounced in small-scale cells and inside hot upflows.

6) The brightness of simulated granules linearly increases with their size for small granules and is approximately constant or even decreases slightly for larger granules. For intergranular lanes the simulations predict a decrease of their brightness with increasing size. It falls very rapidly for narrow lanes and remains unchanged for broader lanes.

7) A quantitative comparison of the brightness properties of simulated granulation with real observations shows that the strong size-dependence of the properties of the smallest simulated granules is not accessible to current observations due to their limited spatial resolution. The observed size dependences result rather from spatial smoothing and the granule-finding algorithm. We do not exclude, however, an influence of the limitations of the 2-D treatment of thermal convection on the present results.

Key words: hydrodynamics - convection - Sun: granulation - photosphere

\section{Introduction}

A large number of fully nonlinear 2-D and 3-D simulations of compressible convection has been carried out to better understand basic features of solar (stellar) thermal convection. They can be roughly divided into two basic groups according to their aims and the detail way they treat physical processes (Nesis et al. 1992). 
The models of the first group were developed to study the major physical mechanisms underlying convectively unstable solar or stellar envelopes. This includes, among others, the simulations of Hurlburt et al. (1984, 1986), Cattaneo et al. (1989, 1990, 1991), Malagoli et al. (1990), Rast \& Toomre (1993a, 1993b), Rast (1998). Although they cannot be used for direct comparisons with observations, their simplicity allows individual physical processes to be isolated and investigated in detail (compressibility, turbulence, ionization effects, topology of convective flows, their transport properties, etc.).

The basic aim of the second group of models is the numerical reconstruction of the complicated structure of solar or stellar atmospheres and their envelopes, including a more or less detailed description of radiative transfer and ionization effects in a compressible, stratified, turbulent medium. These models can be applied to the simulation of spectral lines and reproduce numerous observational properties of solar granulation such as spectral line widths, line shifts, and bisector shapes (Dravins et al. 1981, 1986; Nordlund 1984; Steffen 1989, 1991; Steffen \& Freytag 1991; Gadun 1986; Atroshchenko \& Gadun 1994; Gadun et al. 1999; Asplund et al. 2000b); the distribution of granule sizes (Wöhl \& Nordlund 1985; Gadun \& Vorob'yov 1996); the evolution of granules (Rast et al. 1993; Rast 1995; Ploner et al. 1998, 1999); the reversal of the thermal contrast between granules and intergranular lanes in the photosphere (Nordlund 1984; Steffen 1989; Gadun et al. 1999); correlations between various atmospheric and spectral line parameters (Gadun et al. 1997), and localized anomalies probably related to shocks (Solanki et al. 1996; Gadun et al. 1997). They were used to determine abundance of chemical elements on the Sun (Atroshchenko \& Gadun 1994; Gadun \& Pavlenko 1997; Asplund et al. 2000a; Asplund 2000).

The basic aim of the present project is a numerical study of the size-dependent properties of 2-D thermal convection carried out for the conditions of the solar photosphere and the underlying superadiabatic zone. The main advantage of $2-\mathrm{D}$ simulations is a rapid gain and thus a thorough insight into the effects of varying free parameters and boundary conditions.

For this purpose, we have computed two sets of 2-D models. The first treats thermal convection as roll-like quasi-stationary flows, which are similar to those constructed by Nelson \& Musman (1978) and Steffen et al. (1989). These models are relatively free for problems of large influence of wave-oscillating phenomena on their size-dependence properties, particularly for small-scale cells. The second treats solar convection as a fully non-stationary phenomenon and is a case of more realistic modeling. We have simulated convective and oscillatory motions in a domain with a horizontal size of about $18000 \mathrm{~km}$. Using both sets of models we examine the roles of ionization, radiative transport effects, stability of the flows and their transport properties, concentrating mainly on their size dependence. Finally, we compare the theoretical and observed brightness fields seen as granulation to interpret the observed size dependence of granular brightness. We show here that large scatter in size-dependent properties of small granules can be real because they reflect their various evolutionary history.

\section{Two-dimensional hydrodynamics}

The computational method and the boundary conditions used here to simulate 2-D granular convection were described in the papers of Gadun (1995), Gadun \& Vorob'yov (1996), Gadun \& Pavlenko (1997), and Gadun et al. (1999). One set of models analysed here has $512 \times 58$ mesh-points in the horizontal and vertical directions, respectively, and a spatial step of $35 \mathrm{~km}$. Thus the model domain has $17.850 \times 2.030 \mathrm{Mm}$, since the cells at the right and left model boundaries are used to treat the lateral boundary conditions. The model atmosphere occupies about $800 \mathrm{~km}$ at the top of the model. The time step was $0.3 \mathrm{~s}$ and a total of 71600 time steps, corresponding to 5 h 58 min of solar (hydrodynamic) time, were calculated. Of these the last 5 hours were analyzed.

The calculations were carried out with open upper and lower boundary conditions and periodical conditions at the lateral boundaries. The frequency dependence of the continuum absorption coefficient was included in 97 frequency intervals when treating the radiative energy transport. Absorption due to atomic lines was also considered in the frame of the ODF (opacity distribution function) approach by Kurucz (1979). To solve the system of hydrodynamic equations we used the large particle method (Belotserkovskiy \& Davydov 1982). In order to conserve computational resources we first periodically extended the fully relaxed models with a smaller spatial domain and periodic lateral conditions (they had $114 \times 58$ mesh-points) in the horizontal direction by introducing small variations of the input parameters into the extended model to ensure asymmetric solutions. This was used as the initial condition for the present simulations. Since the simulated solar convection is fully unstable, with interacting flows at different scales, we call them multi-scale models (ms models) in the follows.

To better examine scale-dependent properties of simulated granulation we have also computed a grid of singlescale steady-state models, each with a different horizontal size of the computational domain (hereafter called singlescale or ss models). Their scale-dependent properties are significantly less suffer from wave-oscillating effects and evolutionary history. This is particularly important for subsequent analysis of small-scale inhomogeneous. Their basic parameters are given in Table 1 . The model atmospheres were roughly $500-550 \mathrm{~km}$ deep in all the models. Note that it is mainly the restricted horizontal extent of the domain which leads to the less turbulent, quasistationary flows of these models, since the treatment of 
Table 1. Computed single-scale steady-state models

\begin{tabular}{lllll}
\hline N & $\begin{array}{l}\text { Grid-points, } \\
\text { hor. } \times \text { ver. }\end{array}$ & $\begin{array}{l}\text { Step, } \\
\mathrm{km}\end{array}$ & $\begin{array}{l}\text { Size, } \\
\mathrm{km}\end{array}$ & Note \\
\hline 1. & $20 \times 164$ & 10 & $180 \times 1620$ & stable \\
2. & $24 \times 68$ & 24 & $528 \times 1584$ & stable \\
3. & $38 \times 60$ & 28 & $1008 \times 1624$ & stable \\
4. & $50 \times 60$ & 28 & $1344 \times 1624$ & stable \\
5. & $62 \times 60$ & 28 & $1680 \times 1624$ & unstable \\
6. & $75 \times 60$ & 28 & $2044 \times 1624$ & unstable \\
7. & $87 \times 60$ & 28 & $2380 \times 1624$ & unstable \\
\hline
\end{tabular}

the physical processes is otherwise very similar to the ms models.

We used a simple grey approach to treat radiative energy transfer for the initial duration of about 1 hour of real solar (hydrodynamic) time, i.e. in the interval before the statistically stable regime of the simulations was reached. The simulations of convective motions in each model domain were continued for another hour beyond this moment, but now including a detailed treatment of radiative transfer. This includes transfer effects in molecular lines within the framework of the ODF tables of Kurucz (1993).

\section{General characteristics of the models}

The analysis of every set of single-scale models was based on about 1 hour of time-sequence computed, using the detailed description of radiative transfer. The temporal step between the analysed models was also $30 \mathrm{~s}$. Note, however, that three models from our list of ss models (Table 1), with computational domain sizes larger than or equal to $1680 \mathrm{~km}$, gave unstable solutions. For this reason, all characteristics of these unstable models were determined during their stable phase, prior to the onset of the instability. Thus, for these models the analyzed time was 30-40 min. The analysis of the ss models is described in Sect.3.1. In Sect. 3.2 we describe the analysis of 5 hours of the timedependent multi-scale simulation, with a $30 \mathrm{~s}$ time step between the studied models.

Note that in the classical sense such terms as granule or intergranular lane can be used only to define "bright" and "dark" elements of the brightness field at the solar surface and cannot be invoked to separate convective flows (below the surface, for instance) according to their properties. However, we can prescribe a number of additional characteristics (temperature, pressure, velocity, etc.) for granules and intergranular lanes, if they are defined inside such locations on the surface where we observe the corresponding brightness patterns and if these additional quantities are found for the level where emergent intensity forms. We define the (solar) surface as the mean continuum level, i.e. at $\log \overline{\tau_{\mathrm{R}}}=0$, where $\overline{\tau_{\mathrm{R}}}$ is the Rosseland optical depth averaged over a horizontal level and over the whole time of simulation.

In the model analysis we shall discern between the so called "full" averages of model parameters (which correspond to an average over the whole horizontal extent of the simulation) and means computed separately over upand downflows. Both are determined for a given geometrical level by averaging model data over the total simulation time. The mean parameters of up- and downflows are computed separately for those horizontal positions which are occupied by rising or sinking gas. We have also computed extreme values of the physical parameters of upand downflows at a given horizontal level.

We additionally introduce two classes of quantities to characterize the spatial variations within up- and downflows. They will be called 1) excess or deficit (of temperature, density, etc.), determined as the difference between mean values in up- or downflows and the corresponding "full" average; and 2) the maximum excess or deficit of a parameter if we study the difference between its extreme value and the "full" average.

\subsection{Stable convection: Single-scale models}

Single-scale models describing steady-state thermal convection are presented here. They can be briefly characterized as a treatment of granular convection in which

- convective flows are quasi-stationary in time. Moreover, convection cells do not change their horizontal size in the course of their evolution;

- convection rolls (overturning cells) of any studied scales always extend to the largest depth allowed by the computational box (about $1100 \mathrm{~km}$ below the surface in the present models). This means that granular convection is not a surface (shallow) phenomenon in this approach. Rather, its properties are determined by upflowing thermal plumes. Partial exceptions are the unstable ss models (Table 1 ), which are discussed in detail later.

In spite, or possibly because of their simplicity, the ss models can help us to understand some key results obtained from more realistic multi-scale models mostly because their basic size-dependent properties are less affected by wave and oscillations. Namely, they permit us to

- determine scale-dependent properties of synthetic granulation in a more direct manner;

- study the problem of the instability of the upflow in connection with the sizes of the convection cells;

- estimate contributions of surface effects (i.e., formation of new granules at the surface due to fragmentation of large-scale thermal flows) into observed granular pattern.

Steady-state solutions like the ss models presented here were earlier obtained also by Nelson \& Musman (1978) and Steffen et al. (1989). 

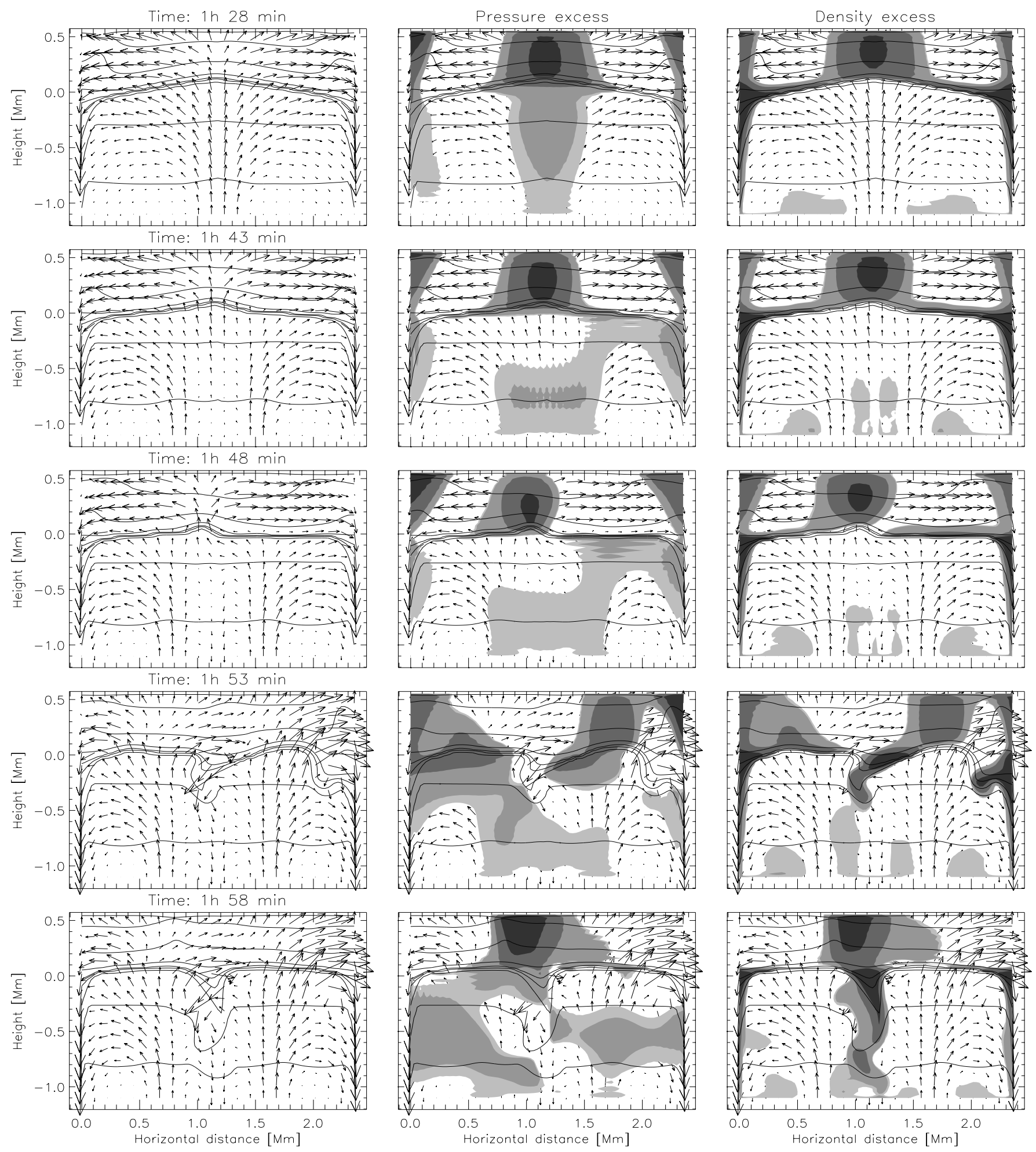

Fig. 1. Development of an instability in the central upflow of a convection cell with a size of $2380 \mathrm{~km}$. The velocity field, isotherms, and regions with pressure and density excesses are shown at five instances during the cell's evolution (from top to bottom). The horizontal lines denote isotherms (from bottom to top): 14 000, 12 000, 10 000, 8000, 7000, 6000, 5000, and 4500 K. Relative pressure $(P / \bar{P})$ and density $(\rho / \bar{\rho})$ excesses above $1.001,1.01,1.1$, and 1.3 at any horizontal level are indicated by shading 


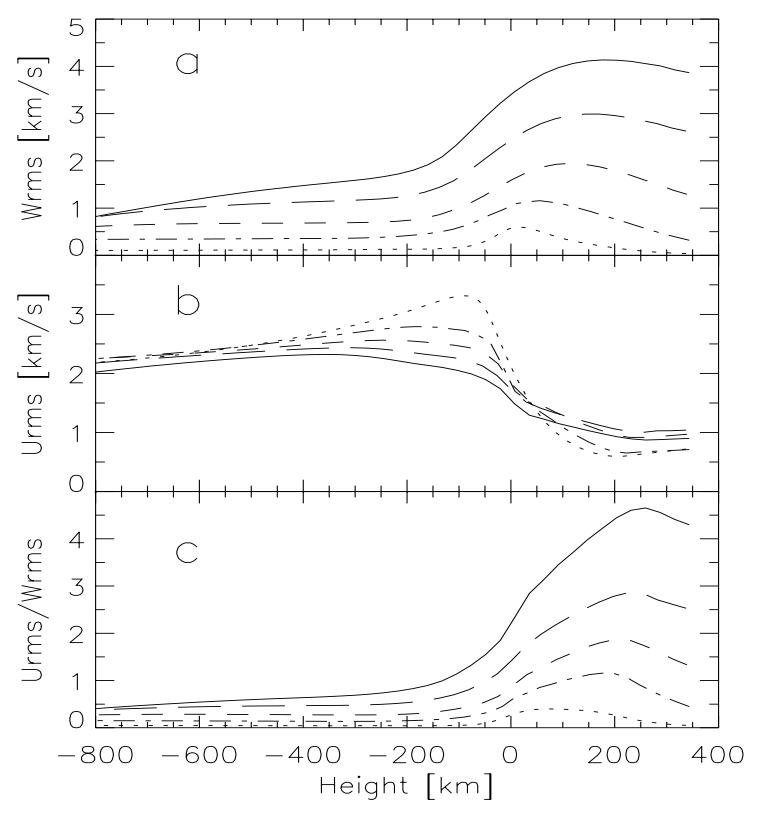

Fig. 2. a) rms horizontal velocities, b) rms vertical velocities, and c) their ratio, shown for five ss models: dotted lines are models with horizontal size $180 \mathrm{~km}$, dash-dotted lines represent models with size $528 \mathrm{~km}$, dashed lines are convection cells with a width of $1008 \mathrm{~km}$, long dashes are those of $1680 \mathrm{~km}$, solid lines are the largest models, having a width of $2380 \mathrm{~km}$

\subsubsection{General evolution}

Our model grid consists of 7 ss models with horizontal sizes of the computational domain ranging from $180 \mathrm{~km}$ to $2380 \mathrm{~km}$ (Table 1). Three models, having horizontal sizes 1680,2040 , and $2380 \mathrm{~km}$, are unstable. Their convection cells fragment in a similar way as that shown in Fig. 1. Figure 1 illustrates the velocity field, isotherms and regions with pressure and density excesses at various instances during the evolution of model with horizontal size $2380 \mathrm{~km}$. Fragmentation starts due to a weakening of the central flow which occurs as a result of the force exerted by the pressure gradient there (buoyancy braking, Spruit et al. 1990 and references therein). Based on these calculations we can note that it has the largest impact in layers lying deeper than the zone of high convective instability (it is discussed later). Figure 1 shows gas can still move upwards inside the high instability zone, although in deeper layers a downflow has already developed.

Among our ss models we distinguish between those of the small cells, which have horizontal sizes smaller than about $800-900 \mathrm{~km}$, the large cells, consisting of ss models larger than $\sim 1500 \mathrm{~km}$, which exhibit unstable solutions, and intermediate cells having, sizes in the range $900-1500 \mathrm{~km}$. The reasons of such a division will become clear in the next section.

\subsubsection{Velocity field}

In Fig. 2 the rms horizontal and vertical velocities as well as their ratio are plotted versus height for five ss models. Peak velocities and other quantities are plotted in Fig. 3 as a function of convection cell size. Figure 3a displays maximum values of rms horizontal and rms vertical velocities and Figs. 3b and c show the peak up- and downflows, respectively. We also represent the minimum fractions covered by downflows (Fig. 3d - "area" filling factor).

Figure 4 reflects the heights at which the peak velocities are found: given in Fig. 4a are photospheric heights at which horizontal velocities reach their maximum values (cf. Fig. 3a). The depths at which the up- and downflow maximum, plotted in Figs. 3b and c, are located are represented in Figs. $4 \mathrm{~b}$ and $\mathrm{c}$.

As follows from Figs. 2a and 3a the horizontal velocities are highly sensitive to the horizontal size of convection cells $L$ (see Steffen et al. 1989). For instance, maximal photospheric rms horizontal velocities are strongly dependent on $L: U_{\text {rms,max }} \sim L^{0.86}$ and are systematically shifted into higher photospheric layers for larger flows (Fig. 4a), from $20 \mathrm{~km}$ to $180 \mathrm{~km}$ above the surface for the cell sizes studied here. This behaviour is explained due to mass balance. These results are compared with those of Steffen et al. (1989) in Sect. 5.

The vertical velocities are less sensitive to the cell size, except for small cells. The general tendencies in the behaviour of vertical velocities are:

- in convectively unstable subphotospheric layers smaller cells exhibit larger rms vertical velocities (Figs. 2b and $3 \mathrm{~b}$ );

- in the photosphere three layers can be recognized (Fig. 2b):

i) a region in which the subphotospheric velocity distribution still dominates (up to $\sim 50 \mathrm{~km}$ above the surface);

ii) a region (from 50 to $100 \mathrm{~km}$ ) where the vertical velocities are nearly cell-size independent;

iii) a region in which the vertical velocities produced by small-scale convective flows become several times smaller than the velocities of cells larger than $1000 \mathrm{~km}$. These possess very similar vertical velocities there.

Granular convection is characterized by geometrical asymmetry between up- and downflows (Cattaneo et al. 1989; Spruit et al. 1990; Hanslmeier et al. 1991). Below we examine differences in properties between up- and downflows, again concentrating on their dependence on cell size.

According to Fig. 3b the largest upflow velocities are nearly constant over a wide range of cell sizes from 500 to $2400 \mathrm{~km}$ and they reach their maximal values near the surface (Fig. 4b): the larger the cell size the closer to the surface this happens (at depths of $60 \mathrm{~km}$ to $20 \mathrm{~km}$ below the surface). 

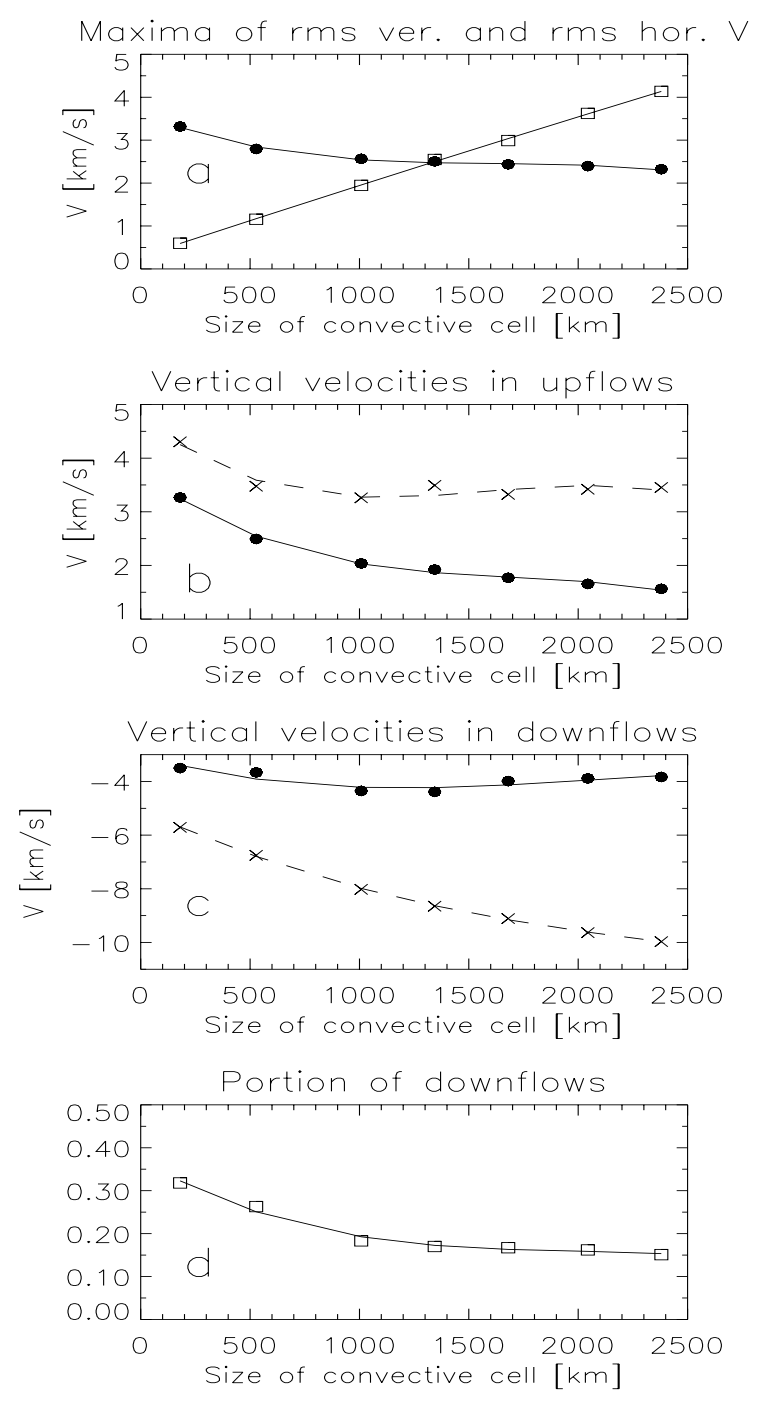

Fig. 3. Dependence of extreme properties of vertical and horizontal velocities on convection cell size. a) Maximum of photospheric rms horizontal velocities (squares) and maximum of rms vertical velocities (dots) in subsurface layers. b) Horizontally averaged (solid line) and the largest (dashed line) vertical velocities in upflows. For each model the velocities are plotted for the layer at which they are the largest. c) Same as frame b, but for downflows. d) Minimum values of "area" filling factors of downflows

It also follows from Fig. $3 \mathrm{~b}$ that the mean velocities of horizontally averaged upflows possess a more obvious dependence on the cell size - larger flows have smaller averaged upflows. Moreover, they attain their maximum values (Fig. 4b) at deeper levels for larger flows (from $70 \mathrm{~km}$ to $100 \mathrm{~km}$ below the surface). Hence, the general tendency for upflows is: small-scale flows have larger mean vertical velocities.

Figure 3c shows that the largest downflow grows rapidly with increasing cell size and (Fig. 4c) is reached
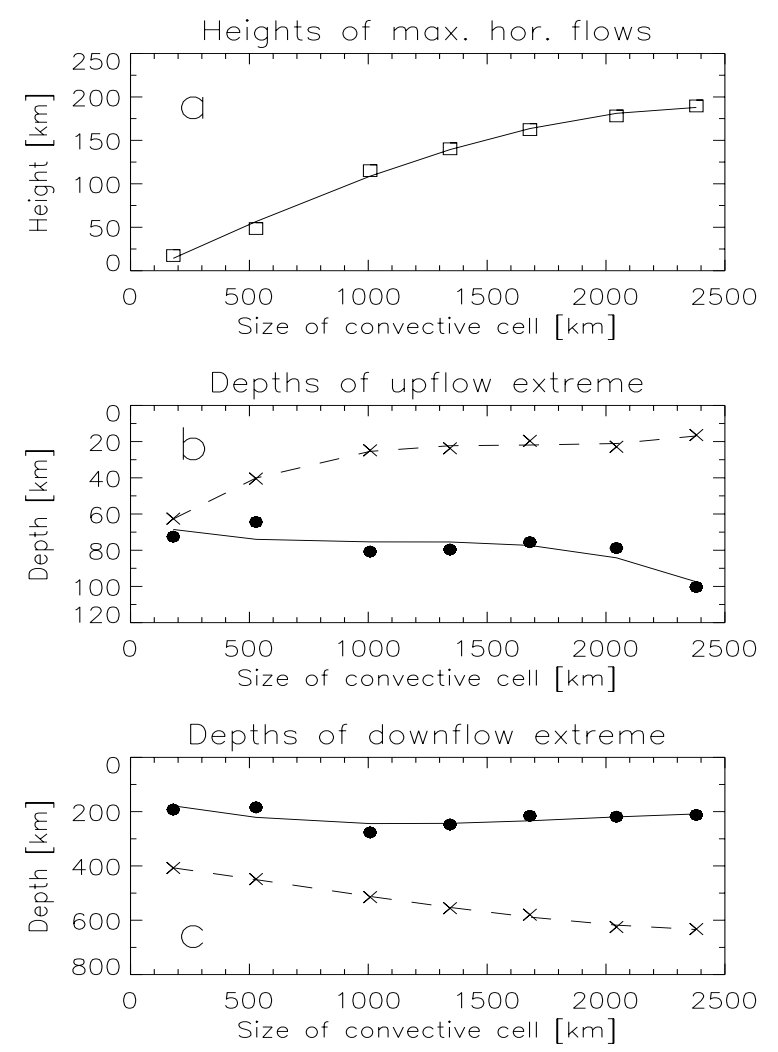

Fig. 4. Heights and depths at which the largest up- and downflow velocities are reached as functions of cell size. a) Height of maximum photospheric horizontal velocity. b) Depths (below the surface) at which the maximum vertical velocities in upflows are located: for horizontally averaged flows (solid line) and for the largest speeds (dashed line). c) The same as b) but for downflows

at increasing depth: from $400 \mathrm{~km}$ to $550 \mathrm{~km}$ below the surface.

However, horizontally averaged downflow velocities (also shown in Fig. 3c) are relatively independent on cell size. Also, these velocities are largest roughly at the same depth $\sim 200-300 \mathrm{~km}$ below the surface.

The asymmetry between down- and upflows becomes less pronounced for small-scale cells. This is seen from the comparison between their absolute up- and downward directed velocities (Figs. $3 \mathrm{~b}$ and c), which lie closer to each other for small cells, as well as from the filling factors of sinking flows (Fig. 3d). In Fig. 3d we plot the minimum fractions of downflows as a function of cell size. As expected for more symmetrical flows the filling factor goes to 0.5 for smaller cells.

\subsubsection{Thermodynamic quantities}

Modeled stratifications of temperature, pressure, and density are shown in Fig. 5. In the lower photosphere (heights from 0 to $+200 \mathrm{~km}$ ) larger cells (long dashed and solid line, 


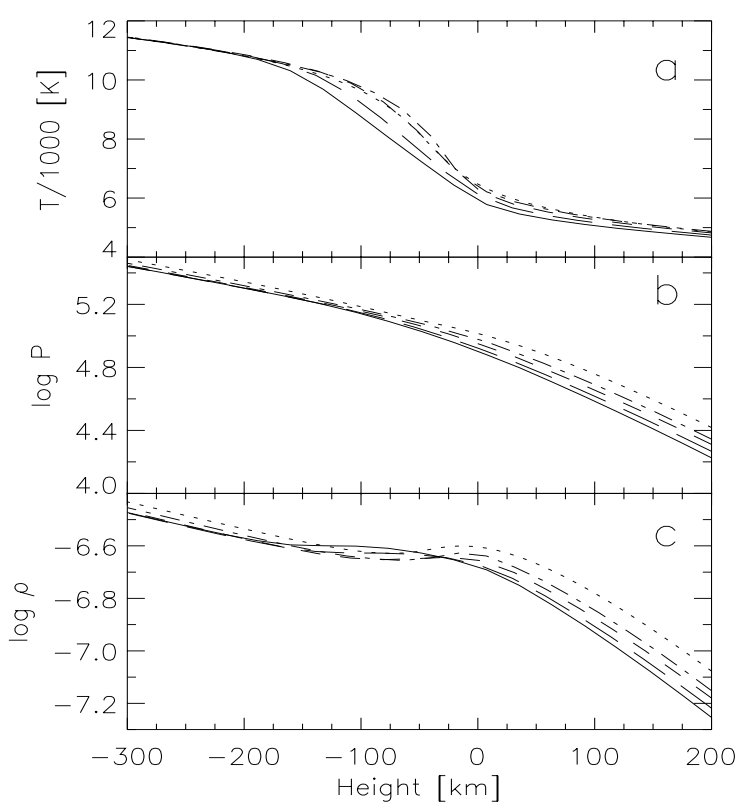

Fig. 5. Spatially and temporally averaged stratification of temperature a), pressure b), and density c) in the five simulations plotted in Fig. 2. Designations of the models correspond to Fig. 2. Pressure and density are given in CGS

cf. Fig. 2) have lower temperature, pressure, and density. However, below the surface, at a depth range from 50 to $150 \mathrm{~km}$, the larger cells are denser than small convection cells due to a density inversion which is particularly pronounced in the latter.

The density inversion occurs in almost all the models (the density at the surface is larger or roughly equal to the density at a depth of $150-170 \mathrm{~km}$ ).

Plotted in Fig. 6 are rms fluctuations of model temperature, pressure, and density. As expected to be, in each model the behaviour of temperature fluctuations and density variations in the convectively unstable regions are in accordance with the distribution of the rms vertical velocities (Fig. 2b). In the atmospheric layers the spatial pressure and density fluctuations correspond to horizontal velocities.

Temperature. Some statistical aspects of behaviour of temperature inside computational domains are given by series of figures. Figure 7 shows temperature stratifications of upward- and downward-directed flows, separately. Figure 8 represents dependence of maxima of rms temperature fluctuations on cell size. We give rms temperature fluctuations in the envelope (Fig. 8a) and in the photosphere (Fig. 8b). In Fig. 8c we show those photospheric heights at which atmospheric temperature fluctuations change their sign. Figure 9 exhibits relative temperature excess/deficit in upflow. In Fig. 10a we display peak values of the temperature excess sampled over depth as a function of convection cell size. We give two values: maximum temperature excess of the horizontally averaged

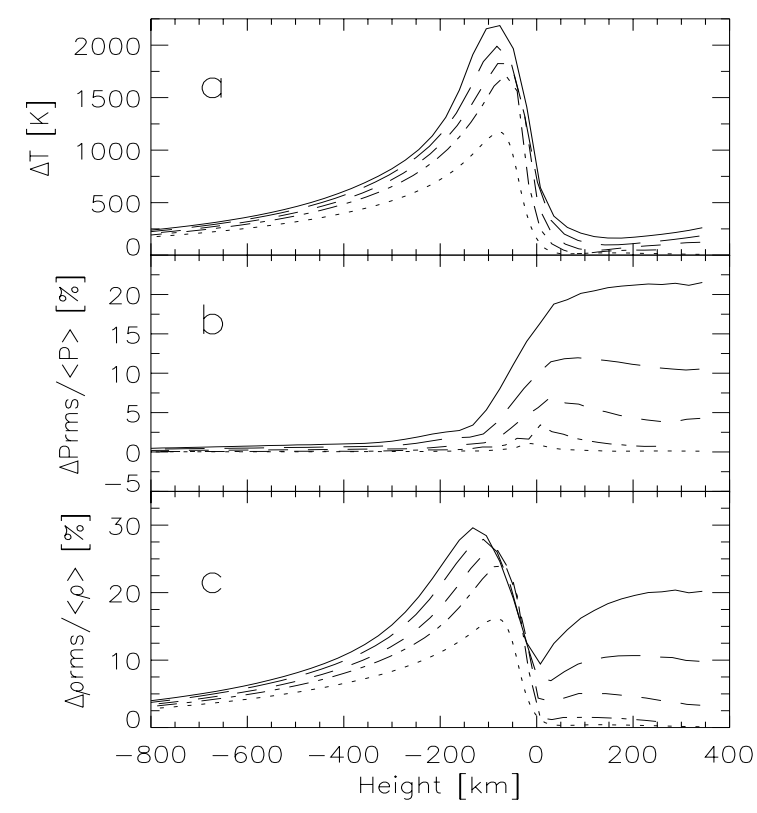

Fig. 6. rms fluctuations of temperature a), pressure b), and density c) of the five ss models as a function of height. Models and designations are the same as in Fig. 2

hot upflow and the largest temperature excess due to the hottest part of the upflow. Both excesses are relative to the "full" average temperature at the same geometrical level. The temperatures in upflows are plotted in Figs. 7a and $b$. The peak values of temperature deficits in downflows are given respectively in Fig. 10b. Their temperature stratifications can be found in Figs. 7c and d. And depths at which these peak values reach their maximum values are shown in Fig. 11a for upflows and in Fig. 11b for downflows.

The main tendencies in size-dependent properties of temperature stratification and temperature spatial variations can be outlined as the follows:

- in small-scale convective cells the temperature gradient below the surface is steeper, on average, than in larger cells (Fig. 7b);

- However, the hottest upflow fragments display the opposite dependence of temperature on cell size: in larger cells the hottest subflows also have the largest temperature gradients (Fig. 7a) which have the tendency to occur at greater heights;

- On average, downflows of larger cells are cooler than those of smaller cells to depths of about $300 \mathrm{~km}$ below the surface. In deeper layers the temperatures of downflows seem to be similar in all the models (Fig. 7d);

- The coolest fragments always show lower temperature for larger cells (Fig. 7c);

- In subphotospheric layers the rms temperature fluctuations are higher for larger convection cells (Figs. 6a and $8 \mathrm{a}$ ). However, this relation is not monotonous. 

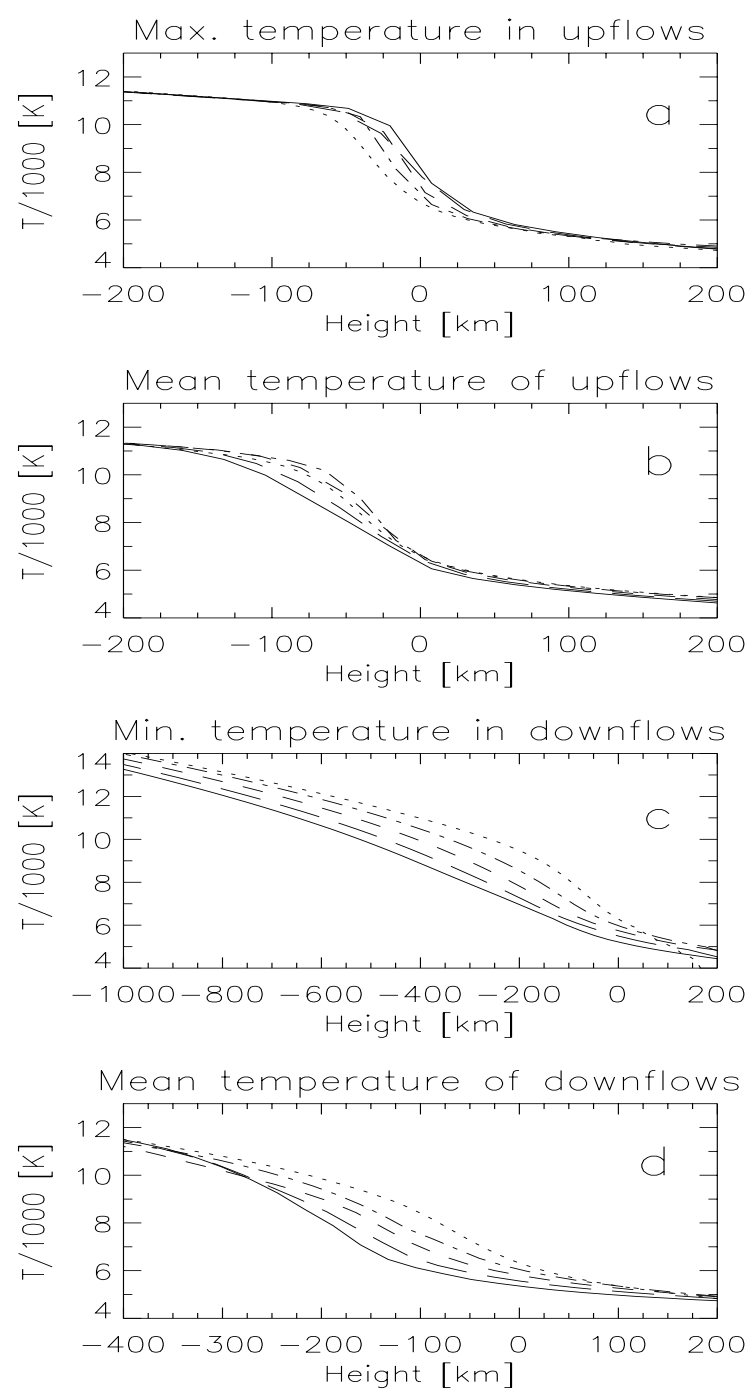

Fig. 7. Temperature stratifications of the five ss models (designations correspond to Fig. 2). a) The hottest fragments in the upflows of convection cells. b) Mean temperatures of upflows. c) The coolest parts of downflows. d) Mean temperatures of the downflows

It differs for cells larger and smaller than about $1000 \mathrm{~km}$. Both groups show this tendency but in cells smaller than $1000 \mathrm{~km}$ the temperature inhomogeneities dissipate faster with decreasing horizontal size (Fig. 8a) than they do for the larger cells;

- As can be seen from Fig. 10a the maximal temperature excess in the horizontally averaged upflow is roughly scale-independent, while its value for the hottest part of the upflow is greater in larger cells. For cells with sizes of about $2400 \mathrm{~km}$ it is 4 times greater than that for the averaged upflow. This suggests that inside large upflows there are hotter portions with properties considerably different from those of the mean flow;

- Below the surface, in downflows the temperature deficit increases with cell size. Moreover, the mean
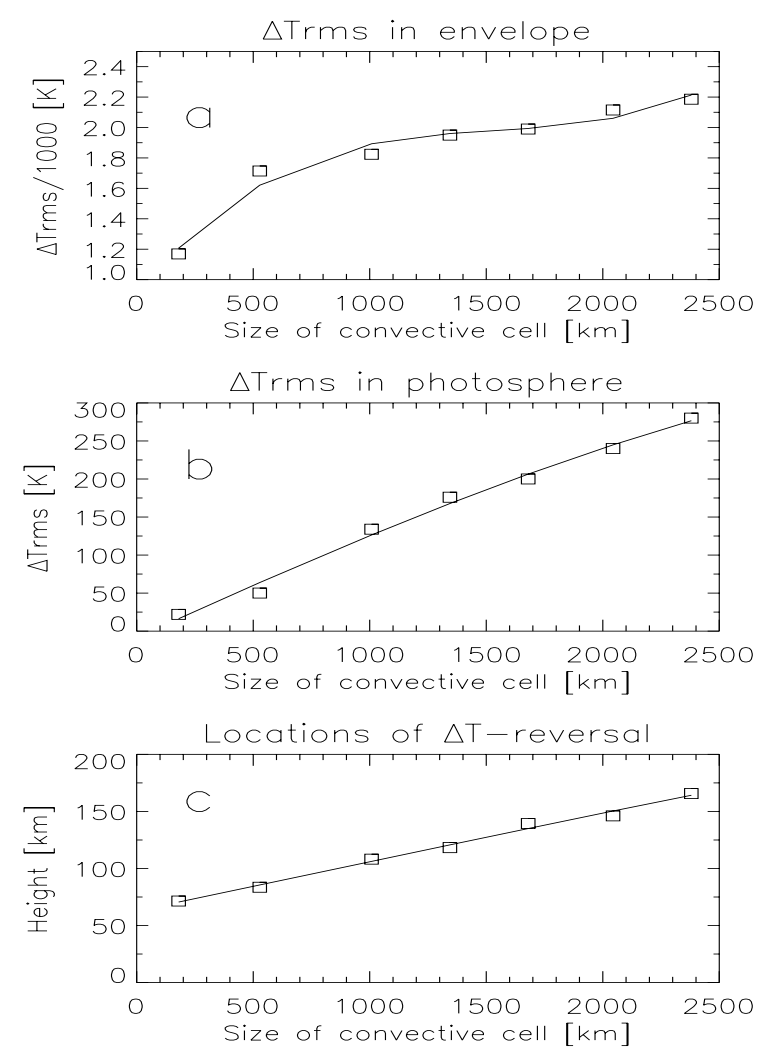

Fig. 8. Size dependence of extreme values of temperature fluctuations. a) Maxima of rms temperature fluctuations in the envelope, i.e. below the surface. b) Maxima of photospheric rms fluctuations. c) Heights at which sign reversals of the temperature fluctuations are found

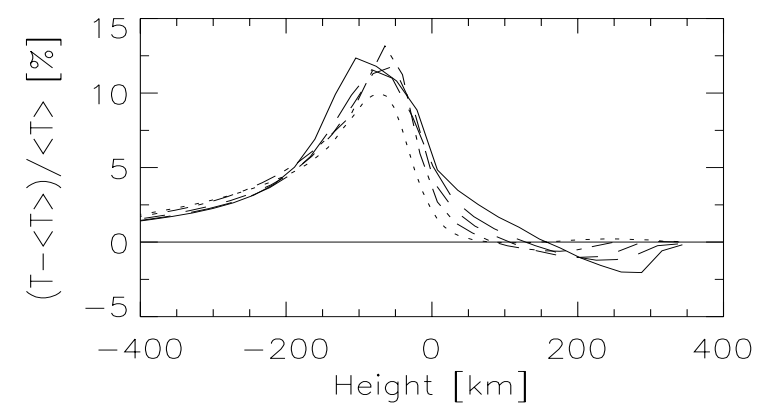

Fig. 9. Relative temperature excess of upflows. Designations of the models are the same as given in Fig. 2

downflows and their parts show similar deficits in dependence on cell size, suggesting that downflows are horizontally more homogeneous;

- In the photospheric layers the larger convection cells show larger temperature fluctuations (Fig. 8b). These values are close to semiempirical estimates made by Altrock (1976), Keil \& Canfield (1978), and Kneer et al. (1980). Moreover, the temperature fluctuations also display a sign-reversal in the photosphere, in 

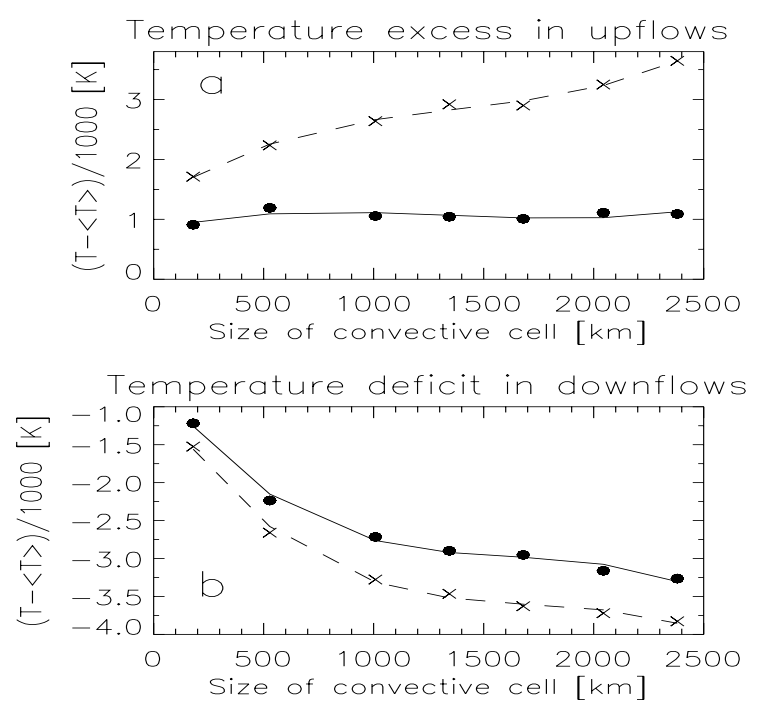

Fig. 10. Size dependence of extreme excesses a) or deficits b) of temperature in up- and downflows. Solid lines are peak values in horizontally averaged up- and downflows relative to the mean temperature; dashed lines are peak values in the hottest and coolest parts of these flows compared with the mean temperature

agreement with earlier investigations (e.g. Nelson \& Musman 1977; Nordlund 1984; Steffen 1989; Steffen et al. 1989; Stein \& Nordlund 1998; Spruit et al. 1990). This is seen in Fig. 9 where the relative temperature excess/deficit in upflows relative to the horizontally averaged temperature is plotted for various ss models. Note how above a certain height the temperature excess becomes a deficit. The geometrical heights at which this occurs are given in Fig. 8c. They vary from 70 to $170 \mathrm{~km}$ depending on cell size.

The depths at which $\Delta T$ of horizontally averaged upflows and those at which the $\Delta T$ their horizontally hottest parts reach their maximum values are shown in Fig. 11a. The depths seen in Fig. 11a coincide with the depth at which the upflows are largest (Fig. 4b). The coincidence between the depths at which downflow velocities and $\Delta T$ in sinking flows reach their extremes is much poorer (Figs. 4c and 11b).

Density. Density stratifications in up- and downflows are given in Fig. 12. For upflows (Fig. 12a) we notice a density inversion for all the cells studied. In contrast, the density inversion, being a result of dynamic compression of the gas, occurs only in downflows associated with the largest convection cells (Fig. 12b).

In Fig. 13 relative amplitudes of horizontal density fluctuations are plotted versus height. The layer, lying just below the surface, in which the convective instability is most pronounced, is clearly discernable (in both upand downflows, Figs. 13a, b). As expected, the density fluctuations have the opposite sign of the temperature
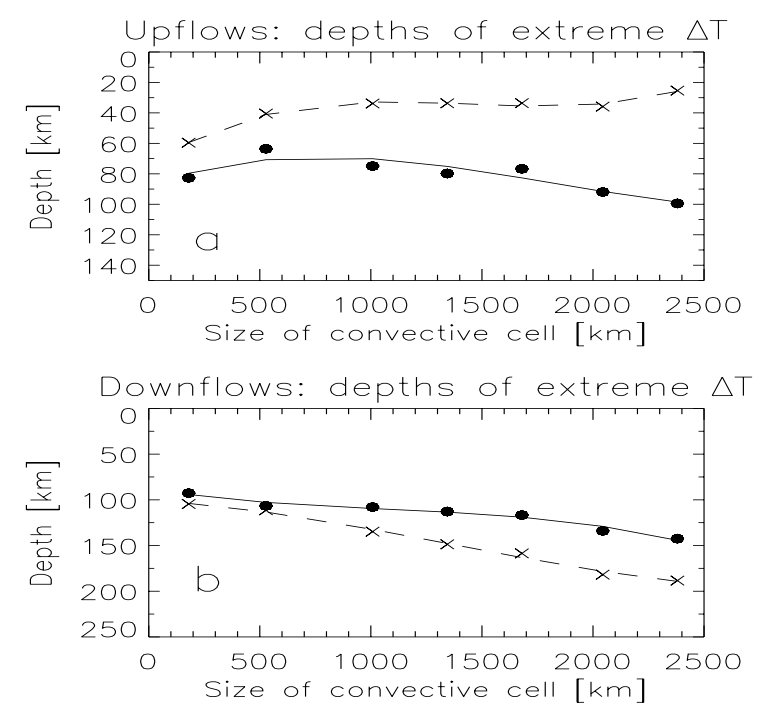

Fig. 11. Depths at which peaks of temperature excess or deficit are located, a) for upflows, b) for downflows vs. convection cell size. Designations are found in Fig. 10
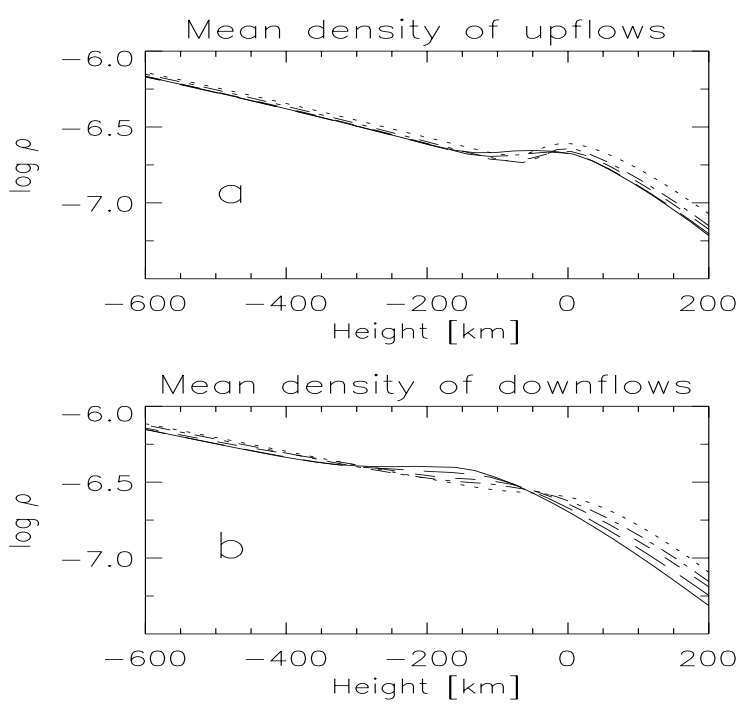

Fig. 12. Density stratification in the models. a) Mean density of upflows; b) mean density of downflows. Densities are given in $\mathrm{g} \mathrm{cm}^{-3}$. Designations of the models are the same as in Fig. 2

variations in the convectively unstable layer and their depth dependence is similar to those found for stratifications of temperature fluctuations.

For instance, the mean densities of upflows, shown in Fig. 13a, exhibit a sharp drop just below the surface, relative to the average over the whole cell. The magnitude of this drop is roughly scale-independent for cells larger than $1000 \mathrm{~km}$ (Fig. 13a). The peak deficits occur at the same depths, at which the peak of $\Delta T$ is localized (Figs. 9, $11 \mathrm{a}$, and 13a). The density deficit of the least dense parts 

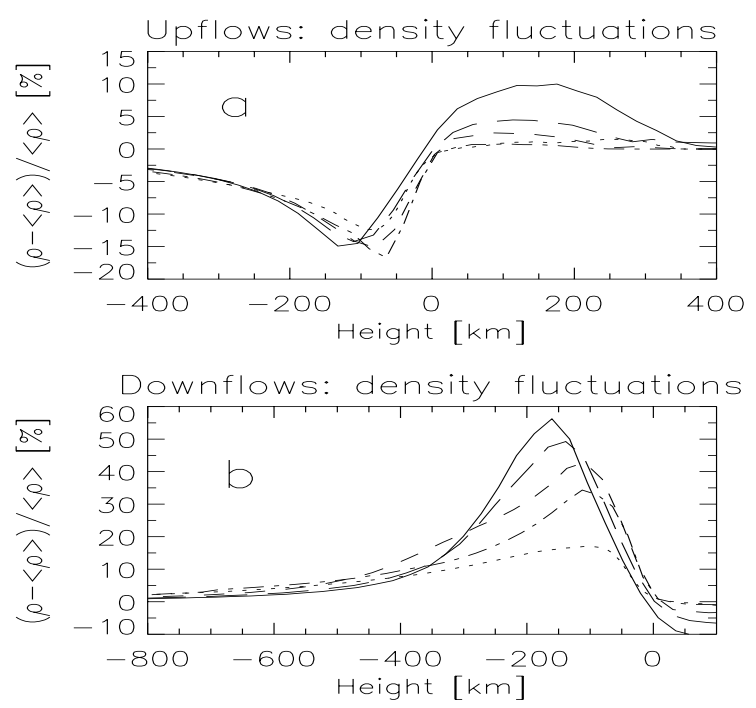

Fig. 13. Amplitudes of density excess (or deficit) in up- and downflows relative to the mean density at a given geometrical level: a) upflows, b) downflows

of upflows increases with cell size in agreement with the growth of the maximum temperature, given in Fig. 10a.

In subphotospheric layers the maximum density excess in downflows, and hence the buoyancy driving in them, grows with increasing cell size. The temperature deficit in downflows exhibit a similar dependence (Fig. 10b). Depths at which the $\Delta \rho$ reach their peaks also agree with the depth at which $\Delta T$ reaches its peak in downflows.

In the low and middle photosphere the density in upflows becomes larger than the average as a result of radiative cooling of hot gas and its compression by the rising flow. This is shown in Figs. 1 and 13a. In contrast, downflows are relatively less dense there (Fig. 13b). Maxima of $\Delta \rho_{\mathrm{rms}}$ in the photosphere depend almost linearly on cell size. The photospheric heights at which $\Delta \rho_{\mathrm{rms}, \max }$ is located decreases slightly for larger cells and lie in the range of 50 to $100 \mathrm{~km}$ above the surface. The height of $\Delta \rho_{\text {rms }, \max } / \bar{\rho}$ increases from $\sim 100$ to $300 \mathrm{~km}$ with growing convection cell size due to a wider peak of $\Delta \rho_{\text {rms }}$ belonging to the largest cells and decreasing density in the photosphere.

Pressure. In compressible convection the pressure fluctuations play a significant role in the evolution of structures (Hart 1973; Massaguer \& Zahn 1980; Hurlburt et al. 1984; Rast 1998). In our simulations $H_{\mathrm{P}}$ (the pressure scale height) changes from about $100 \mathrm{~km}$ at the top of the model, over $\sim 130 \mathrm{~km}$ near the surface, to $450 \mathrm{~km}$ at the bottom of the computational domain. Thus non-local pressure fluctuations cannot be neglected (see Nesis et al. 1999).

Moreover, pressure fluctuations define geometrical topology of convective flows in stratified medium (Massaguer \& Zahn 1980; Hurlburt et al. 1984). Positive
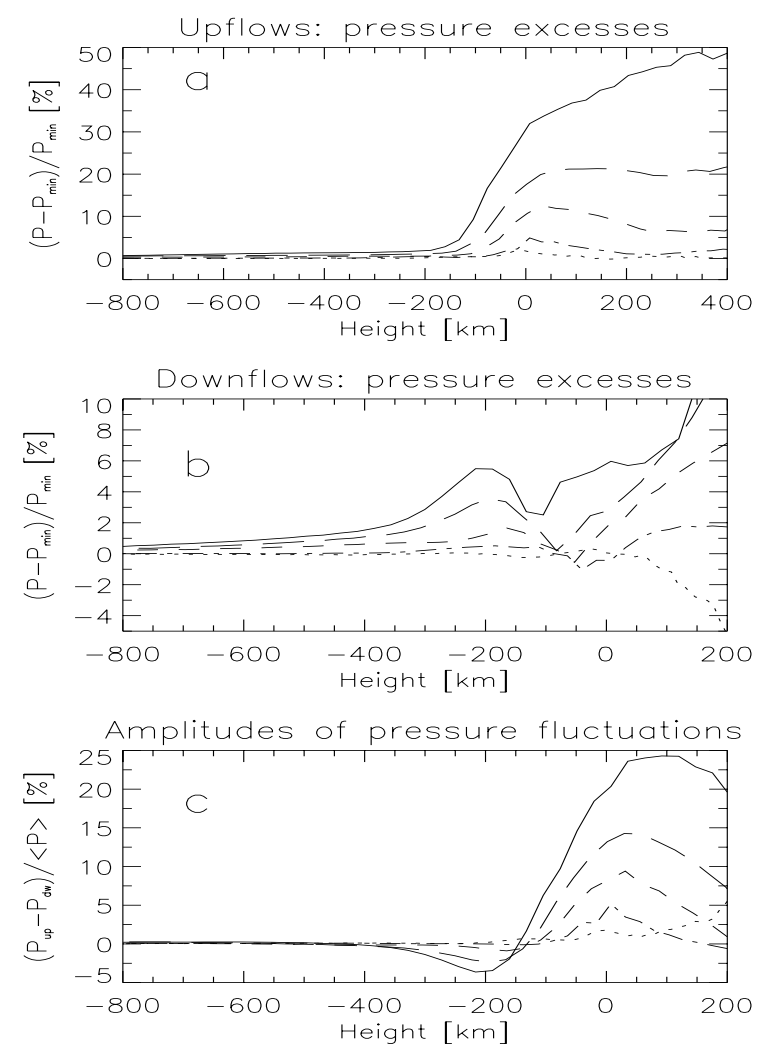

Fig. 14. Height dependence of pressure excesses in up- and downflows. a) Excesses of horizontal mean pressure in upflows are shown relative to the minimal pressure at the same geometrical height, which is located where velocities are nearly horizontal. b) Excesses of mean pressure in downflows relative to the minimal pressure. c) Relative difference between the horizontally averaged pressure in up- and downflows. Designations are the same as in Fig. 2

pressure fluctuations are significant attribute of both: downward- and upward-directed flows. But to do work over long return path of convective flows the pressure inside upflows must be larger than in downflows. Then pressure perturbations increase buoyancy driving of downflows (making them faster and narrower) and build up buoyancy braking in upflows (converting them to broader and gentler structure).

In the ss models the pressure fluctuations are essentially different in layers near the surface, deeper zone of high convective instability located at $100-200 \mathrm{~km}$ below the surface, and in the quasi-adiabatic envelope below it. This can be seen from Figs. 1 and 14 .

In deep layers (starting from $400-500 \mathrm{~km}$ below the surface), on average, the pressure in upflows is larger than in downflows (Figs. 1 and 14c) and the regions of maximum pressure are located in rapid, hot, and rarefied currents (Fig. 1). On an absolute scale the pressure fluctuations increase with depth (in the deep layers) in contrast to the relative values plotted in Fig. 14. From around 


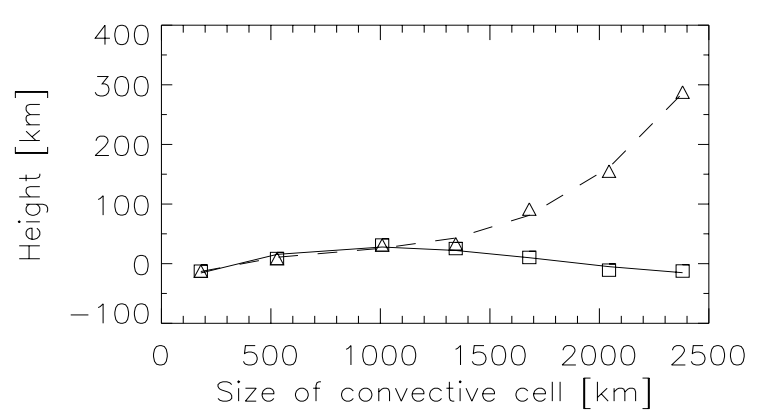

Fig. 15. Height of the largest pressure fluctuations, $P_{\mathrm{rms}, \max }$, in the upper part of the models vs. size of the convection cells. Squares denote the absolute values of the fluctuations; triangles are fluctuations relative to the mean pressure at that height: $P_{\text {rms }, \max } / \bar{P}$

$-150 \mathrm{~km}$ below the surface through at least the lower photosphere the average pressure is considerably larger than in downflows. This is particularly true for the largest convection cells. Note that minimum pressure at a certain horizontal level usually occurs in a region close to the horizontal edge of the upflow where the velocity field is nearly horizontal. In downflows the pressure is always higher than this minimal pressure, with the exception of small-scale cells $(L<600 \mathrm{~km})$. However, relative to the pressure averaged over the whole horizontal extent of the convection cell, the pressure averaged over downflows is often smaller.

Just below the zone of maximum convective instability (see the next section) the steady-state models exhibit an reversal of the pressure fluctuations: on average, the pressure in upflows is lower (Fig. 14c).

In this series of ss simulations we have postulated closed lateral boundary conditions. The closed lateral "walls" of the model domain do not permit the hot rising flows to expand and so to reduce their high pressure. Thus, the downward-directed streams are squeezed much more strongly than can be expected for a periodical treatment of the lateral boundaries. This increases the speed of the sinking gas, reducing their "area" filling fraction, and, finally, leads to the development of inversion of pressure fluctuations.

As can be seen from Figs. 1 and 14a, at the surface very high pressure fluctuations develop above upflows due to effective cooling there (making the surface a thermal boundary for adiabatic convective flows: Spruit et al. 1990; Schüssler 1992; Rast et al. 1993). This excess pressure produces horizontal velocities in the photosphere.

The height at which this $\Delta P_{\mathrm{rms} \text { max }}$ is reached in the photosphere are shown in Fig. 15. We plot two relations: $\Delta P_{\text {rms,max }}$ (squares) and its value normalized to the horizontally averaged pressure (given by triangles). The maximum pressure fluctuations occur at the surface, between the peaks of photospheric density fluctuations and those of temperature.
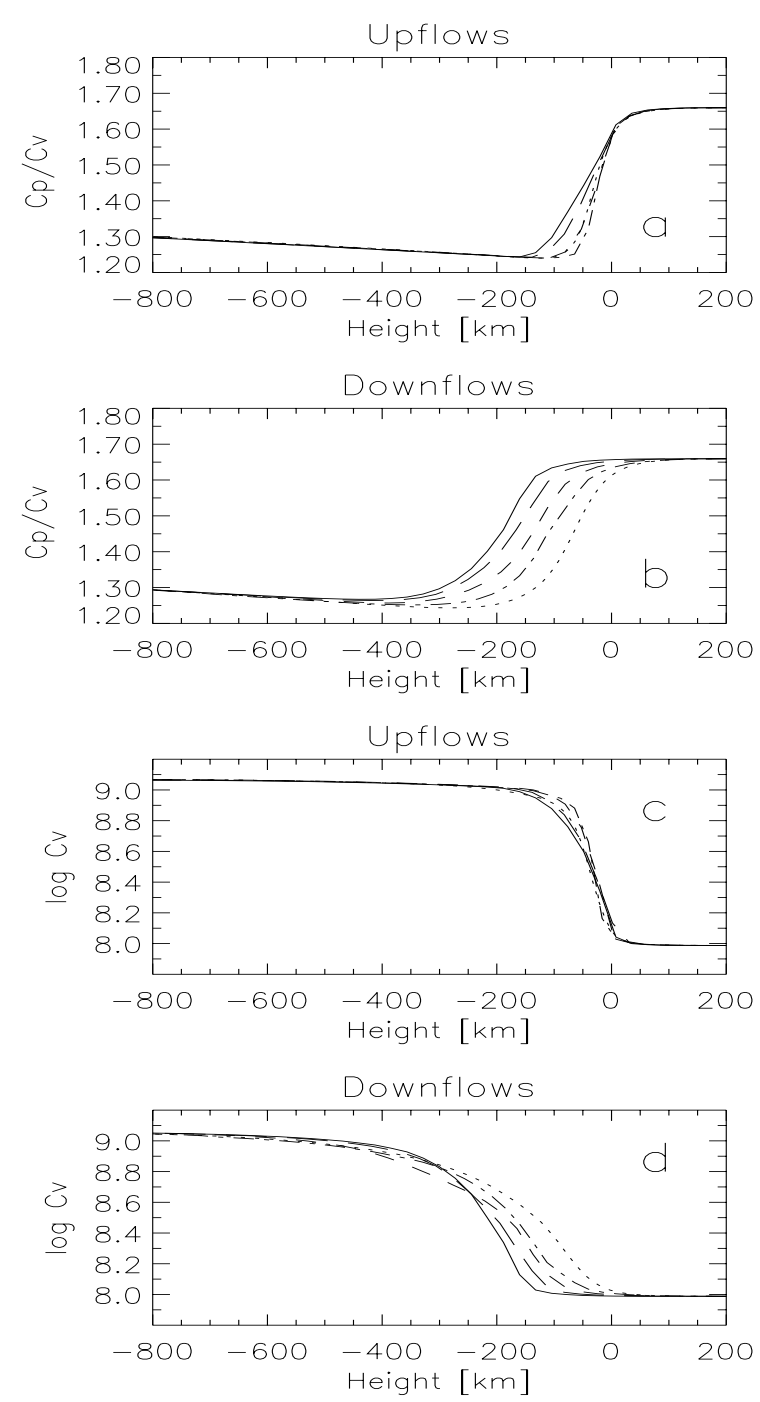

Fig. 16. Stratification of the ratio of specific heat at constant pressure $\left(C_{\mathrm{P}}\right)$ to that at constant volume $\left(C_{\mathrm{V}}\right)$ in up- a) and downflows b), as well as $\log C_{\mathrm{V}}$ in rising c) and sinking d) gas. The curves represent convection cells of different sizes. $C_{\mathrm{V}}$ is in units of $\operatorname{erg~g}^{-1} \mathrm{~K}^{-1}$. Designations are those given in Fig. 2

A pressure excess (at a fixed horizontal level) is also seen near downflows in the photosphere (Figs. 1 and 14b). It directs horizontal velocities downward. But averaged over downflow it is lower than that above upflows.

\subsubsection{Zone of convective instability}

In general, the properties of compressible solar convection are controlled by stratification, radiative heating and cooling (thermal conduction), turbulence, and ionization effects. In this section we focus on layer containing of partially ionized hydrogen in the context of its role in granular convection. 

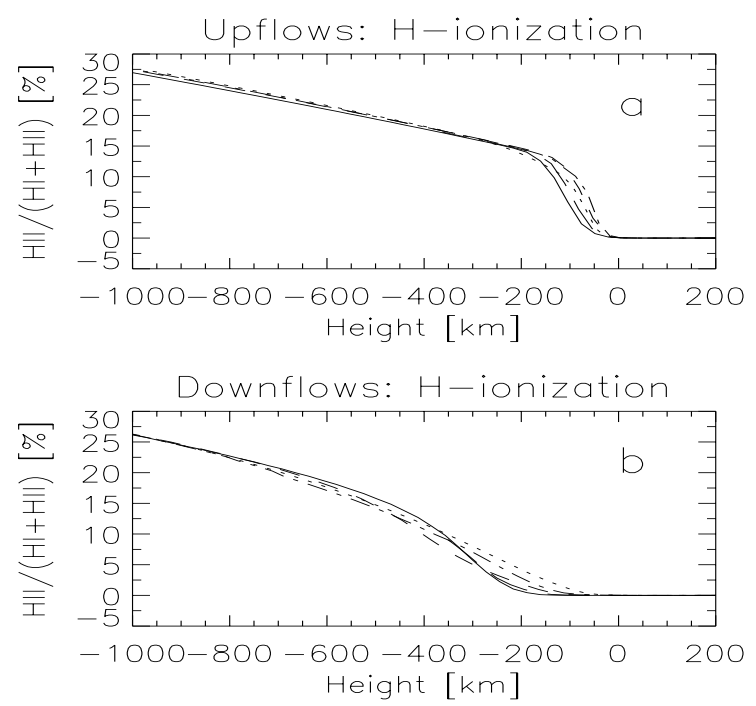

Fig. 17. Fraction hydrogen that is ionized relative to the total hydrogen number density in a) up- and (b) downflows. Designations are the same as in Fig. 2

The partial ionization of hydrogen leads to the development of a pronounced convective instability in the corresponding. It produces clear signatures in the horizontal fluctuations of thermodynamic quantities as well as in the velocities.

Efficient ways in which ionization can influence the basic thermodynamic properties of a gas are: changes of specific heat, opacity, internal energy, and pressure (Rast et al. 1993).

Due to the ionization of hydrogen both the specific heat at constant volume $\left(C_{\mathrm{V}}\right)$ and that at constant pressure $\left(C_{\mathrm{P}}\right)$ grow by an order of magnitude in the depth range from the surface to about $200 \mathrm{~km}$ below it in upflows and down to $400 \mathrm{~km}$ in downflows. This process is illustrated by Fig. 16, where the height-dependence of the ratio $\gamma=C_{\mathrm{P}} / C_{\mathrm{V}}$ and $\log C_{\mathrm{V}}$ are shown for up- and downflows.

In upflows changes in $\gamma$ and the specific heat in upflows depend only weakly on cell size and occur within a narrow depth range. In downflows, however, they change over a broader depth range.

These values can be compared with the fraction of ionized hydrogen, plotted in Fig. 17. It is obvious that the largest changes in $\gamma$ and the specific heat are observed at the top of the region of partial ionization of hydrogen, where about $5-10 \%$ of hydrogen is ionized.

The local pressure gradient is also changed due to changes in particle number density. As a result of recombination, for instance, the pressure is reduced and this accelerates gas to move upwards. The extremes of $\Delta T$, $\Delta \rho$, and $W_{\text {rms }}$ coincide in averaged upflows and are seen at depths from $60 \mathrm{~km}$ to $100 \mathrm{~km}$ below the surface. They do not depend strongly on cell size.
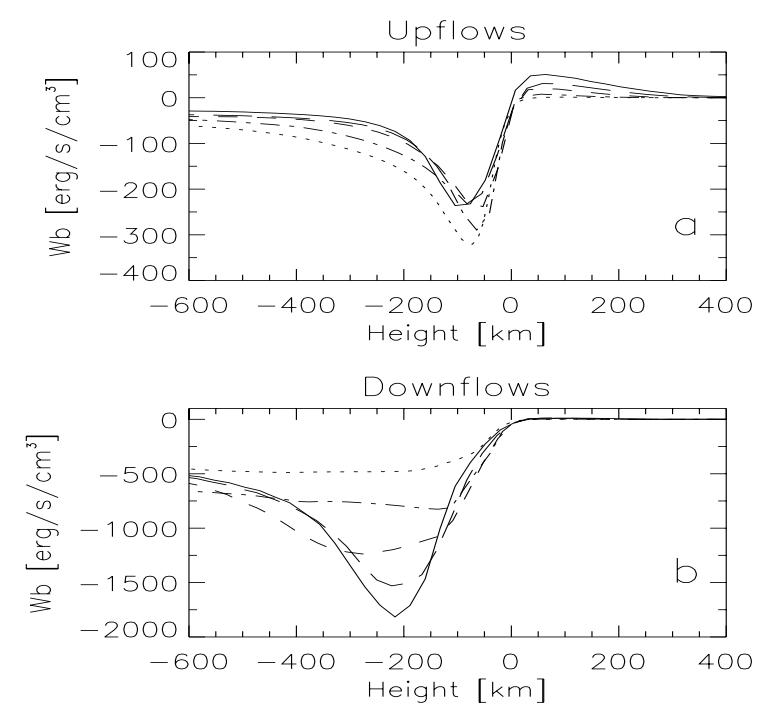

Fig. 18. Height dependence of the work done by buoyancy forces in five ss models plotted separately for $\mathbf{a})$ up- and $\mathbf{b}$ ) downflows. Designations are the same as in Fig. 2

The buoyancy force $\left(W_{\mathrm{b}}\right)$ shown in Fig. 18 was calculated as:

$W_{\mathrm{b}}=g W \Delta \rho$,

where $W$ and $\Delta \rho$ are the vertical velocity and the horizontal density fluctuations, respectively, and $g$ is the gravitational acceleration. For thermal upflows the mean buoyancy force work also depends weekly on their horizontal size, more precisely, for cells larger than $1000 \mathrm{~km}$ it remains roughly unchanged.

We note also the important role of opacity in these layers. The Rosseland mean opacity increases in upflows by a factor of more than 100 from the surface to $100 \mathrm{~km}$ below it (Fig. 19c). The absorption coefficient is most sensitive to the temperature exactly at the same depths, i.e. $50-100 \mathrm{~km}$ below the surface (Fig. 20a). This additionally protects hot flows from radiative cooling. The reason of this strong temperature sensitivity of opacity lies in the high dependence of $\mathrm{H}^{-}$absorption on electron density. Above a depth of $50 \mathrm{~km}$ the photon mean free path in large-scale upflows (displayed in Fig. 19a) becomes larger than $50 \mathrm{~km}$ and they are cooled actively.

The extremes of internal energy variations are located at the same depth as those of the temperature and density fluctuations. However, these relative fluctuations are larger, for instance, by a factor of 2 with respect to relative temperature fluctuations due to ionization effects.

In summary, the zone of high instability can be easily localized in upflows: its location corresponds to that at which the most pronounced peaks of $\partial C_{\mathrm{V}} / \partial z, T$-excess and $\rho$-deficit occur. Moreover, several effects work together so as to increase the convective instability of upflows in the zone of partial ionization of hydrogen. They lead to a significant growth of the buoyancy force there, 

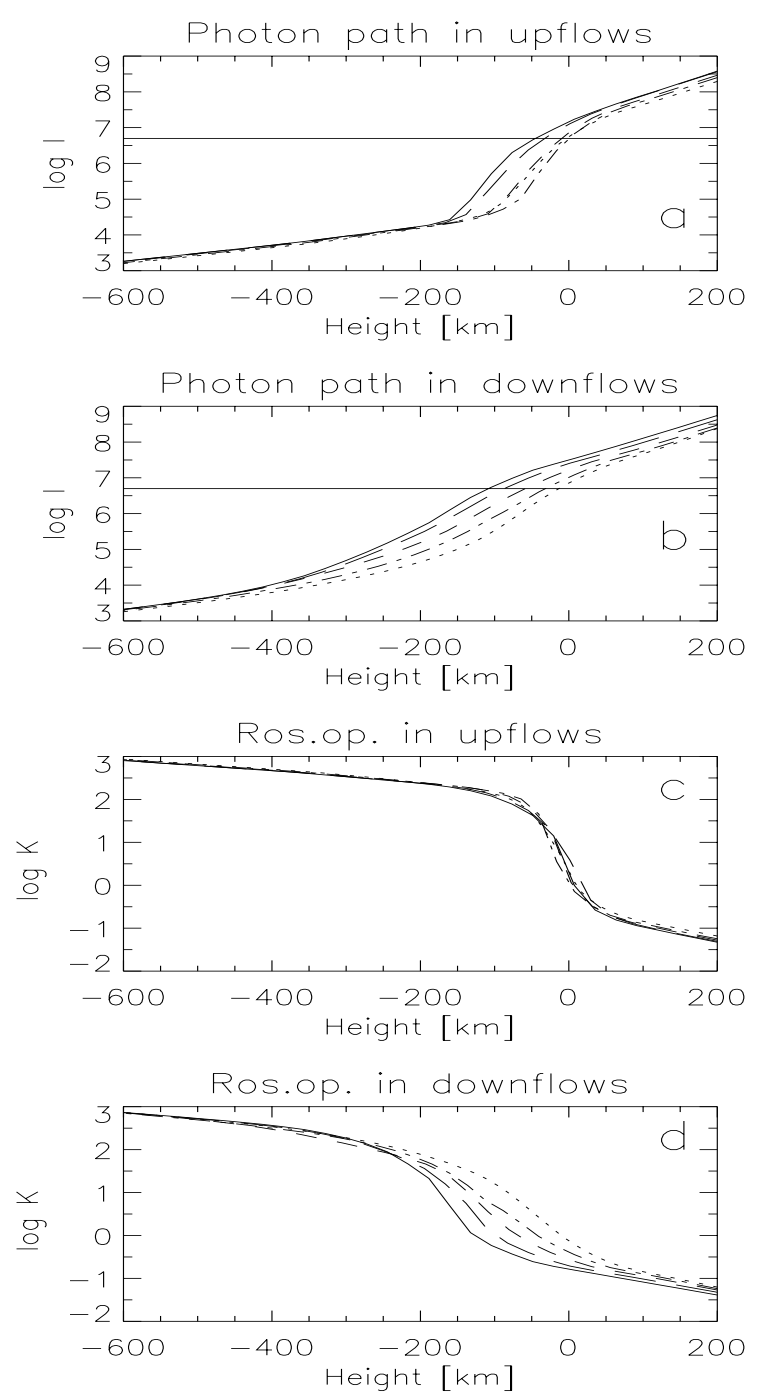

Fig. 19. Stratification of the photon mean-free path and Rosseland opacity for up- and downflows in the models. Designations of the models correspond to those shown in Fig. 2. Horizontal solid lines in a) and b) correspond to a photon mean-free path of $50 \mathrm{~km}$. The photon mean-free path is computed using the Rosseland mean opacity and is given in $\mathrm{cm}$. The Rosseland opacity is in units of $\mathrm{cm}^{2} \mathrm{~g}^{-1}$

so that just below the surface there is a narrow layer which increases convective instability.

The dynamics of downflows differ in essential aspects from those of upflows. The growth of the fraction of ionized hydrogen takes place in deeper layers (Fig. 17b) as compared with the levels where the maxima of $T$-deficit and $\rho$-excess are observed (Figs. $11 \mathrm{~b}$ and $13 \mathrm{~b}$, respectively). The layers at which the largest downward-directed velocities are seen (Fig. 4c), are also shifted with respect to the $\Delta T$ - and $\Delta \rho$-extremes. At last, all downflow parameters (including $W_{\mathrm{b}}$, Fig. 18b) demonstrate sharp and well pronounced dependence on horizontal size of downflows (convective cells). This strong size dependence is explained
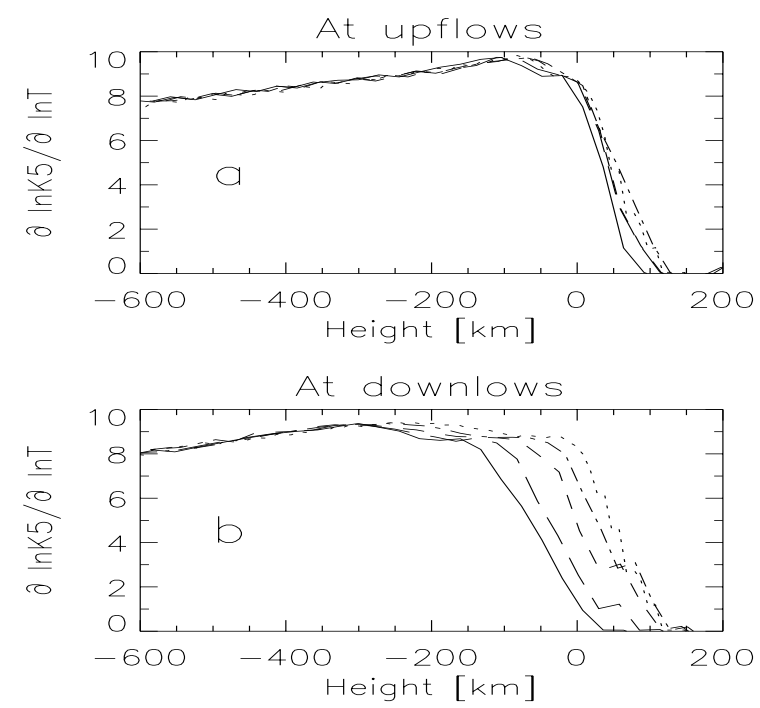

Fig. 20. Temperature sensitivity of the monochromatic opacity coefficient at $\lambda 500 \mathrm{~nm}$ averaged over up- a) and downflow b). Designations are the same as in Fig. 2

by sensitivity of their buoyancy driving to horizontal radiative heating (Steffen et al. 1989): as broader they are as less effective is their radiative heating.

It is important, for understanding of dynamics of downdraughts, that the increase of thermal damping in downflows due to horizontal radiative heating near the surface occurs before ionization effects change thermodynamic properties of the gas. The ionization effects inhibit this process and maintain energetic downflow plumes. This follows from the stratification of the Rosseland opacity (Fig. 19d), photon mean freepath (Fig. 19b), temperature sensitivity of the monochromatic absorption coefficient at $500 \mathrm{~nm}$ (Fig. 20b) and is in contrast to upflows where ionization effects increase the instability of upflows before they are influenced by radiative cooling.

\subsubsection{Continuum intensity fluctuations}

In Fig. 21 we show relative rms fluctuations of emergent monochromatic intensity at $\lambda 500 \mathrm{~nm} . \Delta I_{\mathrm{rms}} / \bar{I}$ exhibits a similar dependence on convection cell size as $\Delta T_{\mathrm{rms}}$ (discussed above and plotted in Fig. 8a): around $L \sim$ $1000 \mathrm{~km}$ (this corresponds to "granules" in the size range of $500-600 \mathrm{~km}$ ) it is seen that the dependence on $L$ changes. We also plot the $\Delta I_{\mathrm{rms}} / \bar{I}$ resulting from largescale models during the unstable phase of their evolution. Such values are represented in Fig. 21 by triangles and show that large cells exhibit only marginally smaller intensity fluctuations in this case.

Based on the scale dependence of the intensity fluctuations, these convection cells can be divided into two groups, those with sizes smaller and larger than $\sim 1000 \mathrm{~km}$, 


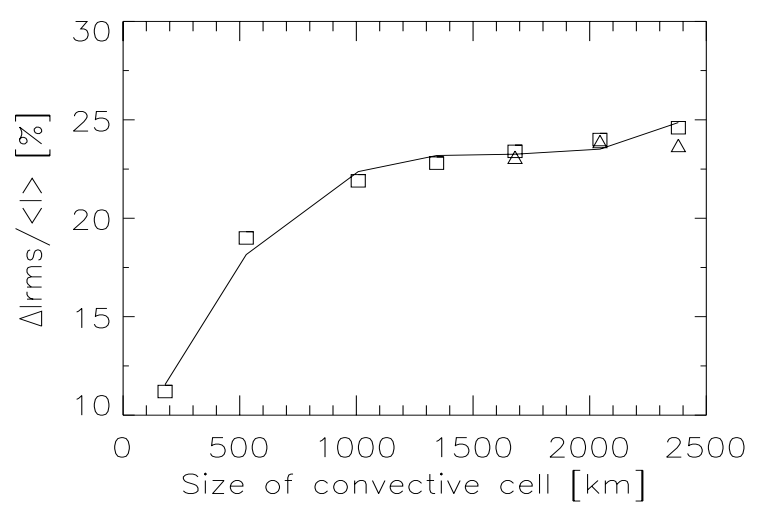

Fig. 21. Relative rms fluctuations of continuum intensity at $\lambda 500 \mathrm{~nm}$ plotted vs. convection cell size. For large-scale cells two values are shown: those corresponding to stable (squares) and unstable (triangles) solutions

respectively. For the first group radiative damping plays a very active role and intensity fluctuations decrease rapidly with decreasing cell size. For the second group $\Delta I_{\text {rms }} / \bar{I}$ is virtually independent of $L$.

These results are in good qualitative agreement with the detailed study of Steffen et al. (1989). Quantitatively, our models exhibit higher intensity fluctuations by about a factor of 2 .

\subsection{Non-stationary convection: Multi-scale models}

The multi-scale models are free from most of the main assumptions underlying the ss models, in particular those outlined in Sect. 3.1. A typical snapshot of the velocity field, isotherms, areas with supersonic flows, and regions having pressure and density excesses in the model are displayed in Fig. 22. The regions with large Mach number (associated with shocks) can be divided into the following 3 categories, examples of each of which are found in Fig. 22:

- supersonic oscillations of the vertical velocity localized in the upper part of the model atmosphere, near and above the traditional temperature minimum (e.g., between horizontal locations $x=(6-9) 10^{3} \mathrm{~km}$ in Fig. 22);

- supersonic horizontal granular velocities situated in the middle photosphere (e.g. near $x=10^{4} \mathrm{~km}$ );

- supersonic downflows near and under the visible surface (at $x=2000 \mathrm{~km}$ in Fig. 22).

Often the downflows of oscillating motions exceed the local sound speed in the upper atmosphere. Moreover, they interact with overshooting convection by increasing the granular horizontal velocities. As a consequence, the maximum Mach numbers of vertical oscillations and the maximum Mach numbers of horizontal velocities of overshooting convection oscillate fairly in phase. Subphotospheric supersonic downflows are often caused by the sharp increase of downflows after being pulsed by the impact of horizontal flows at the intergranular lane (Cattaneo et al. 1989). Their Mach number fluctuates approximately in antiphase.

The relative fluctuations of pressure and density reach their maxima in the upper atmospheric layers of the model, where they are caused by a combination of the buoyancy braking mechanism acting on convective flows and the oscillatory motions. Inhomogeneities in these layers are influenced mainly by oscillations. Consequently, their boundaries do not coincide with the spatial distribution of the granular brightness field (Fig. 22).

The temporal evolution of 2-D granules in the current set of ms models has been studied by Ploner et al. (1998, 1999). They show that the evolution of individual synthetic granules ends either through fragmentation or dissolution, in the most cases, depending on their mean sizes. Granules smaller than $1000-1400 \mathrm{~km}$ mainly dissolve whereas the evolution of larger structures is in general terminated by their fragmentation. This result agrees well with the behaviour of the ss models if we take into account that convection cells with a horizontal sizes of $1500-1600 \mathrm{~km}$ produce bright "granules" with sizes of about $1000 \mathrm{~km}$. It is also in good agreement with the observations of Kawaguchi (1980) and Karpinskiy \& Pravdjuk (1998).

\subsubsection{General characteristics of ms models}

The height dependence of temporally and spatially averaged rms vertical and horizontal velocities are shown in Fig. 23, together with filling factors of up- and downflows.

We have to note that these models are a case of direct numerical experiment. This means that we did not correct any model quantity (including velocity components) inside computational domain or at the model boundaries. Hence, large values of vertical velocities seen in Fig. 23a are resulted by our treatment of physical processes and/or details of numerical calculations. In particular, extremely large velocities are demonstrated by less dense downflows. Their behaviour depends in large degree on treatment of radiative effects. In contrast to the steady-state models we did not include the contribution of molecular lines to the opacity. This enhanced the dynamics in the ms simulation. There is also fundamental reason in producing large model oscillations because in simulations the very limited computational domain is normally used compared with the real solar case (Stein et al. 1989). They can be in principal reduced with special boundary conditions (Stein \& Nordlund 1998; Grossmann-Doerth et al. 1998; Gadun et al. 1999).

Figure 24 displays the stratification of averaged rms temperature fluctuations and amplitudes of $\Delta T$ variations, where $\Delta T$ is the temperature difference between horizontally averaged up- and downflows. 

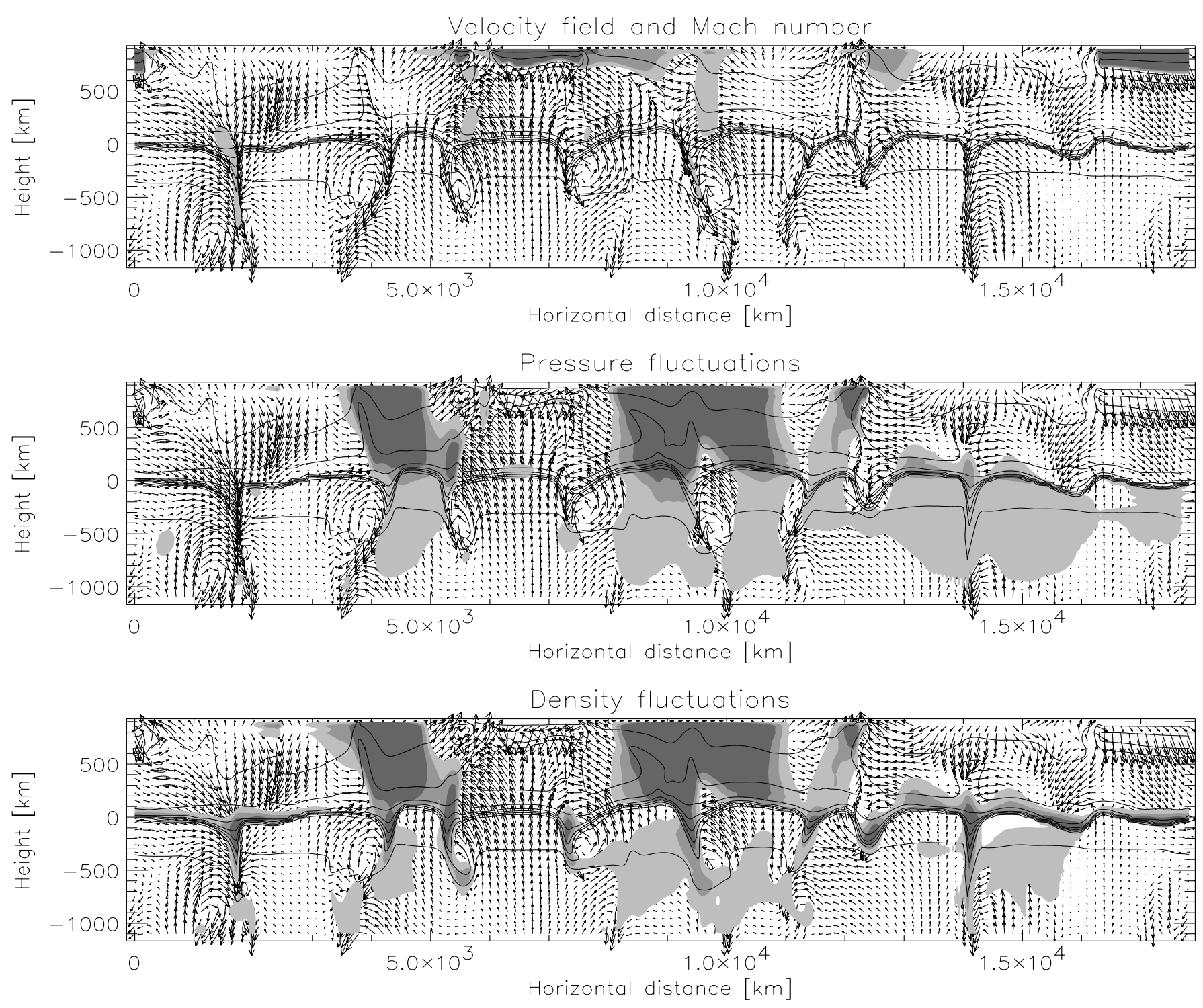

Fig. 22. A representative snapshot of $2-\mathrm{D}$ multi-scale models. Arrows represent the velocity field. Horizontal lines are isotherms (from bottom to top): 12 000, 10 000, 9000, 8000, 7000, 6000, 5000, and $4000 \mathrm{~K}$. Mach numbers above 0.9, 1.2, and 2.0 are denoted by different strengths of the shading in the top frame. Relative pressure $(P / \bar{P})$ and density $(\rho / \bar{\rho})$ excesses greater than $1.02,1.2$, and 1.4 are similarly marked in the middle and bottom frames. The average $\bar{P}$ and $\bar{\rho}$ are determined over horizontal layers

In convectively unstable layers rms vertical velocities, horizontal velocities, and rms temperature fluctuations are close to those found for large-scale ss models. In the upper model atmosphere vertical velocities are higher in the ms models, due to the influence of oscillations. The atmospheric temperature fluctuations are also larger for the same reason.

The reversal of the temperature difference between up- and downflows is seen at $\sim 170 \mathrm{~km}$ above the surface in Fig. 24b. Near the traditional temperature minimum we note a second reversal of the temperature difference: upflows become hotter again. This is due to the in- creasingly large contribution from oscillations in the upper photosphere. Upflows of ms models also demonstrate an inversion of density in subphotospheric layers (Fig. 25), in agreement with the ss models.

Vertical velocities and excesses and deficits of $T, P$, and $\rho$ are given in Fig. 26 for up- and downflows separately. Values averaged horizontally over up- respectively downflows are considered. These results are reasonably close to the corresponding values for the steady-state solutions, although there are also important differences. In upflows, the largest velocity is observed $\sim 120 \mathrm{~km}$ below the surface, at the depth of the maxima of $T$-excess and 


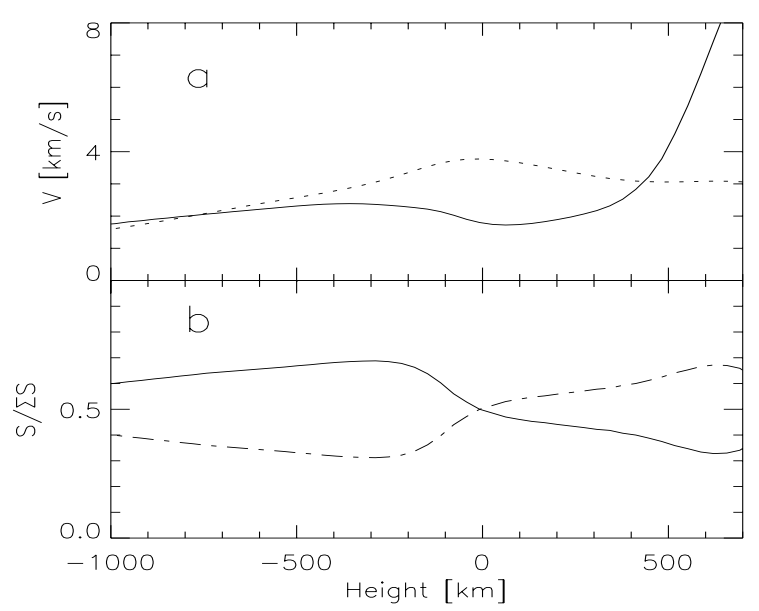

Fig. 23. a) rms vertical (solid line) and horizontal velocities (dots) averaged horizontally and over time versus height and b) "area" filling factors for up- (solid line) and downflows (dotdashed line) also as a function of height. The plotted data refer to ms models

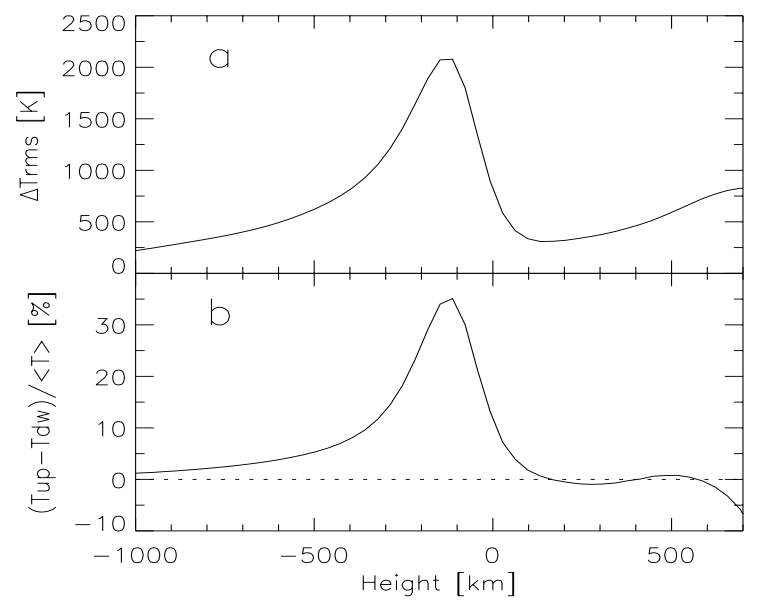

Fig. 24. a) Horizontally averaged rms temperature fluctuations of the ms models versus height and $\mathbf{b}$ ) difference between averaged temperatures in up- and downflows. In b) the dashed line represents the zero-level of the temperature variations

$\rho$-deficit (see Banos \& Nesis 1990). In contrast, in downflows, the largest vertical velocities in subphotospheric layers are shifted to greater depth with respect to the extremes of temperature and density fluctuations (300 $350 \mathrm{~km}$ vs. $200 \mathrm{~km}$ ). The velocity maximum instead roughly coincides with the smallest area occupied by downflows (Figs. 23b and 26d).

In contrast to ss models, in these simulations we do not see a pressure inversion. However, at a depth of $270-350 \mathrm{~km}$ the amplitude of pressure fluctuations decreases (Fig. 26e). It is caused by a strong growth of pressure in downflows (Fig. 26d) and is close in depth to the minimal filling factor of downflows (Fig. 26d). Therefore,

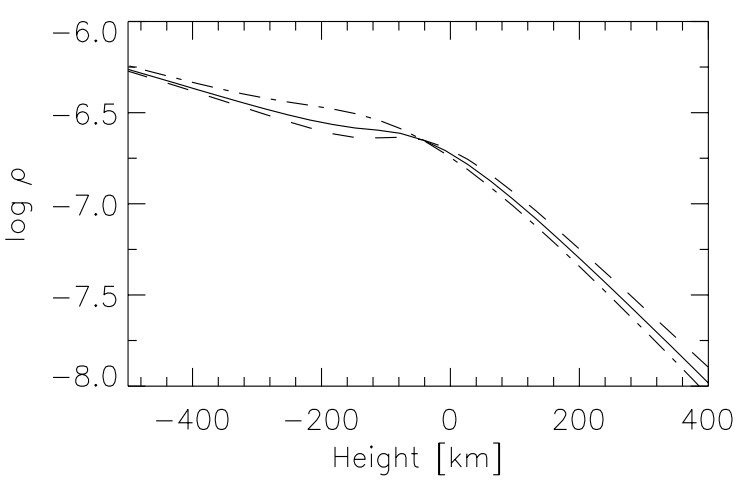

Fig. 25. Height-distribution of ms model density averaged horizontally and over time: mean density (solid), averaged density in upflows (dashed), mean density in downflows (dot-dashed). The density is in $\mathrm{g} \mathrm{cm}^{-3}$

at these depths the rising thermal plumes exhibit the largest horizontal sizes.

\section{Size-dependence of observable properties of 2-D granulation}

In Sect. 3 we aimed to obtain a better understanding of 2-D granulation, in particular the size dependence. Now we consider how the observable physical properties of convection cells in both the ms models and the ss models, depend on their size, which is now represented by the size of the visible granule to be directly compared with the observations.

In accordance with the definition generally used by observers we consider granules to be regions of excess brightness. We have computed the monochromatic emergent intensity at $\lambda 500 \mathrm{~nm}$ at each horizontal location at each time step and have identified granules using isophotes of the monochromatic intensity: a granule is defined as a onedimensional brightness excess above the mean intensity emerging from the simulation at the corresponding timestep. For every granule and intergranular we have also computed a number of physical parameters as described in Sect. 3.

\subsection{Steady-state models}

In the ss models that go unstable, i.e. models with horizontal size of $1680 \mathrm{~km}$ or greater, as the fragmentation starts a part of unstable models is seen to darken until it takes on the appearance of an intergranular lane. It splits up the single brightness excess (single granule) into several smaller brightness fragments which are, for a short time, actually produced by the same broad convective flow. These brightness fragments we shall call the unevolved structures in the following. 

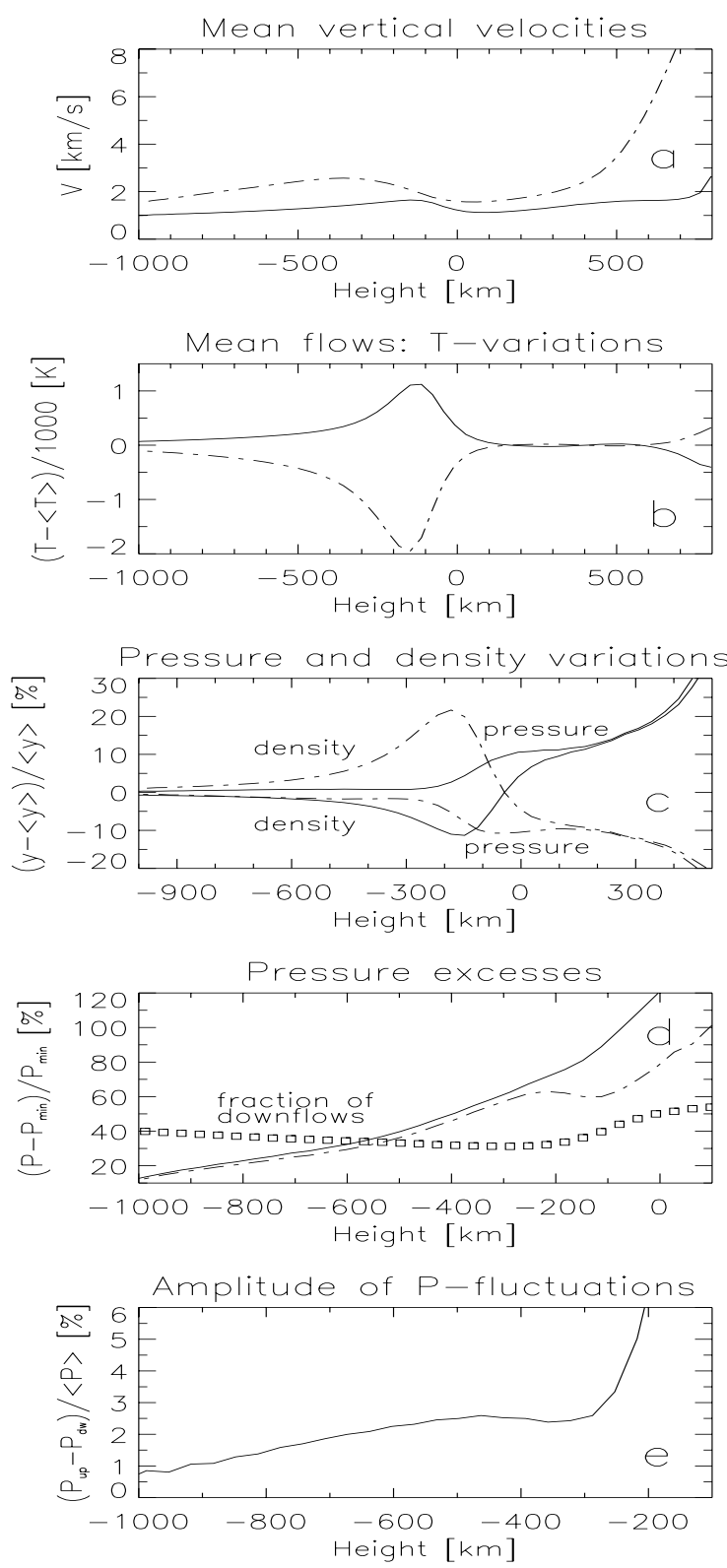

Fig. 26. Stratification of various physical parameters in the ms simulations. a) Stratification of the unsigned vertical velocities averaged horizontally over up- and downflows, b) the difference between the mean temperature in and the average temperature, $\mathbf{c}$ ) relative excess of density and pressure in up- and downflows, d) pressure excesses in up- and downflows with respect to the minimal pressure inside upflow and a fraction of downflows (squares); all the quantities are given in percents, e) relative difference between mean pressures in up- and downflows, a measure of the amplitude of pressure fluctuations. In a-d) the solid lines represent upflows, dot-dashed ones downflows
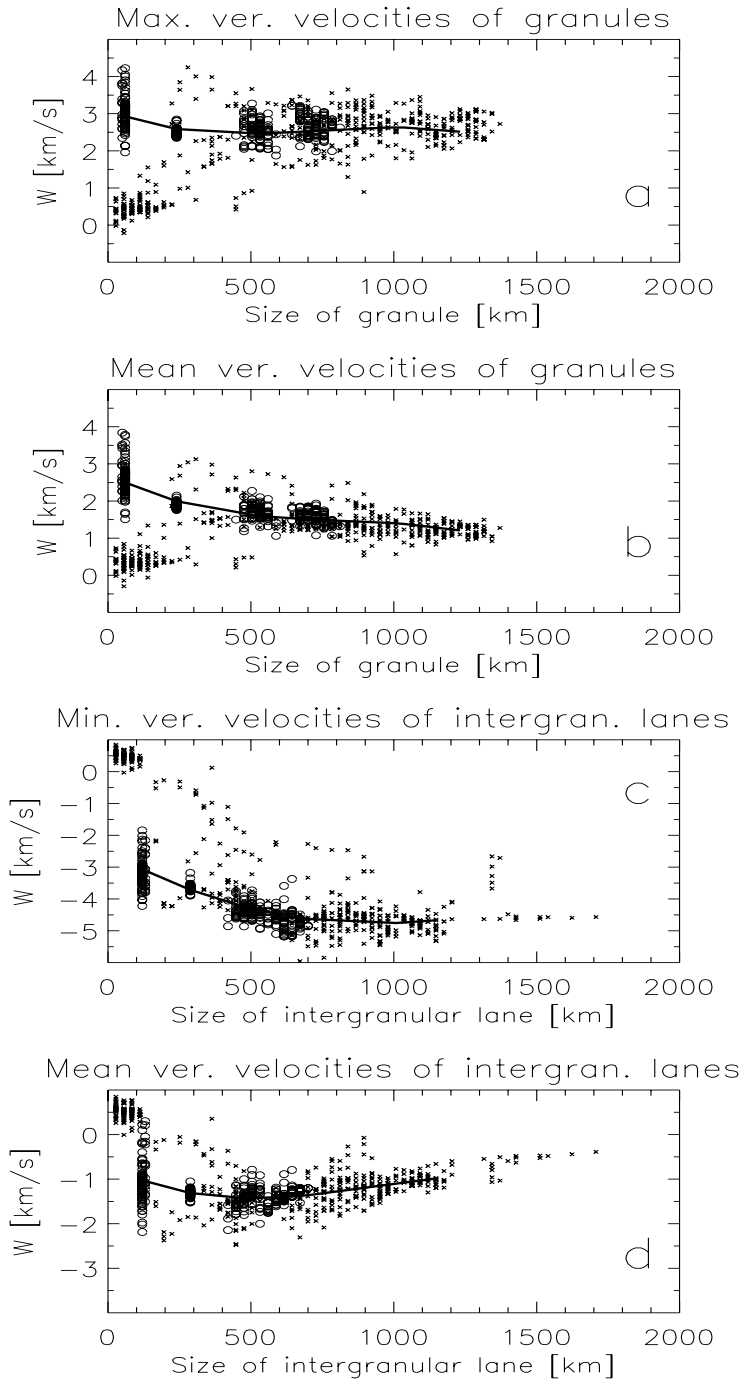

Fig. 27. Maximum a) and mean b) vertical velocities for granules, and minimum c) and averaged d) vertical velocities for intergranular lanes as a function of the size of the granule (a and $\mathbf{b})$, respectively of intergranular lanes (c and $\mathbf{d})$. Circles are stable convection cells, small crosses mark unstable models (fragmenting cells), and solid lines are the mean relation computed only for the stable phase of cell evolution. Plotted are the results at the visible surface $\log \tau_{\mathrm{R}}=0$ of the steady-state models of thermal convection

Shown in Figs. 27 and 28 are the velocities and temperatures of granules and intergranular lanes computed from ss models. We distinguish between stable (represented by open circles) and unstable (crosses) models.

In Fig. 27 the stable and unstable solutions differ essentially in the small-scale range. Stable small-scale flows exhibit larger velocities connected with granules and lower downward-directed velocities associated with intergranular lanes than we found for equally small, unevolved structures in unstable solutions (which are formed during the fragmentation of the original large cell). 

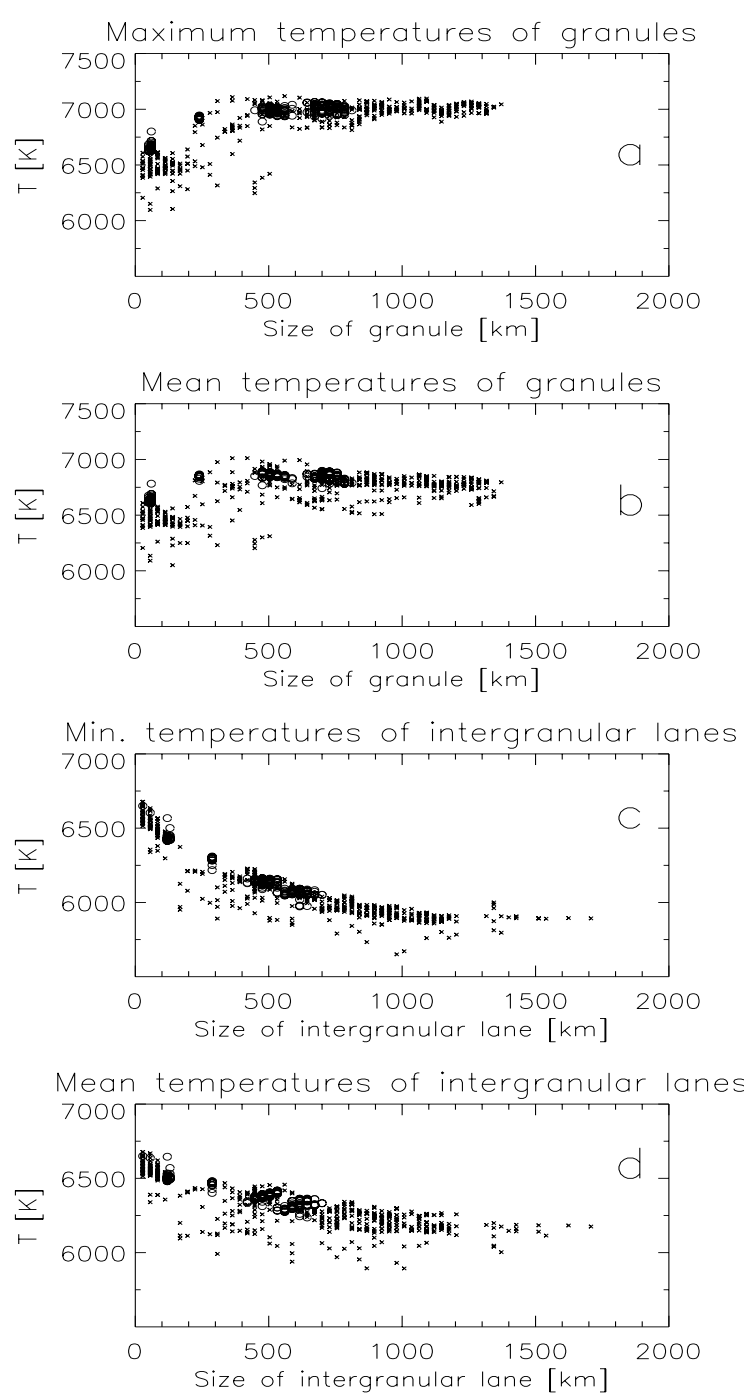

Fig. 28. Scale dependence of maximum temperature a), and average temperature b) of granules, as well as minimum c) and mean d) temperatures of intergranular lanes. The plotted values refer to the surface $\log \tau_{\mathrm{R}}=0$ of steady-state simulations. Designations are the same as in Fig. 27

In Fig. 28, on the other hand, the two populations of small-scale granules almost coincide (although, stable small-scale cells display slightly higher granule temperatures).

The temperatures of intergranular lanes belonging to stable convection cells exhibit the same size dependence as the intergranular lanes produced by the fragmentation of large-scale unstable cells.

In Fig. 29 we plot the intensity of two sets of scans computed for unstable "steady-state" models only. The intensity distributions look similar to the temperature distributions (Fig. 28), with the exception of the mean intensities of intergranular lanes. The fact that this behaviour is not seen in Fig. 28d suggests that the temperature gradient is
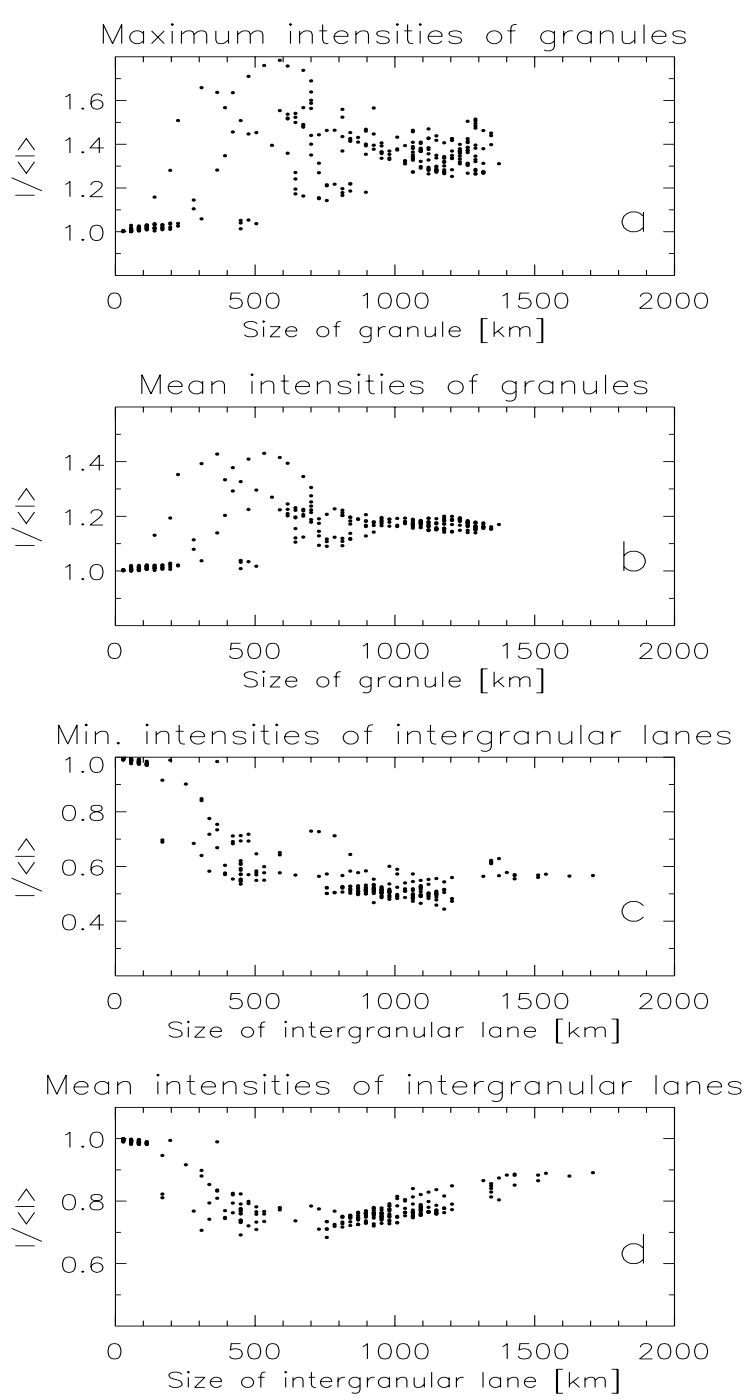

Fig. 29. Dependence of maximum a) and mean b) intensities of granules and minimum c) and mean d) intensities of intergranular lanes on their sizes. Plotted are results of two sets of unstable models which have horizontal sizes 2044 and $2340 \mathrm{~km}$

significantly different for small and large-scale intergranular lanes bordering unstable granules.

So, the nature of thermal convective flows, either as shallow (surface) convection or as a collection of deepformed convection cells, is well defined in the dependence of vertical velocities of small-scale granules on their sizes. But it cannot be recognized in scale dependence of the temperature.

\subsection{Multiscale simulation: Non-stationary convection}

Because ms models produce oscillations with strong amplitudes we have removed the $p$-modes from the studied time-dependent intensity field as well as from the associated variables. The removal technique is described by 

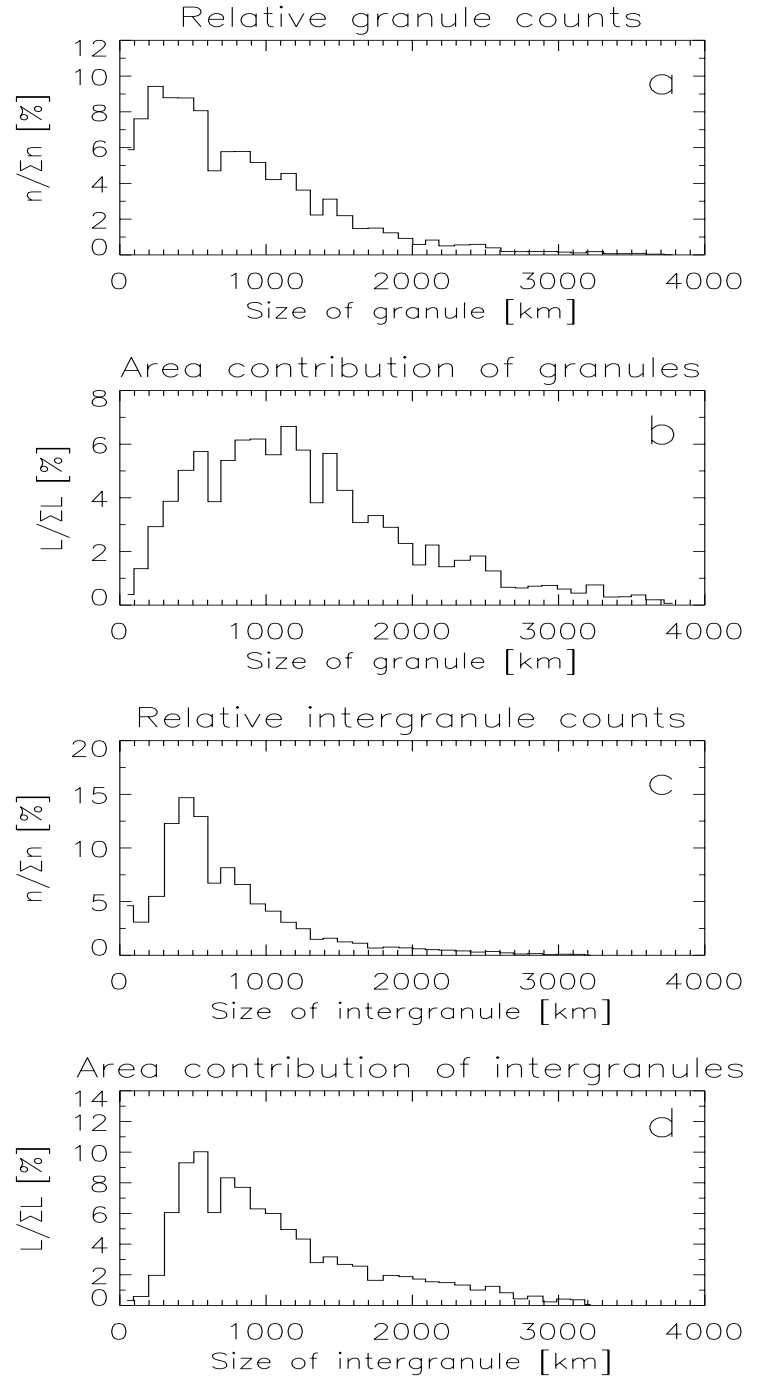

Fig. 30. Histograms of the relative granule count a) and of the contribution of granules to the total "area" covered by granules b) obtained for intensity at $\lambda 500 \mathrm{~nm}$. In c) and d) the same quantities are plotted for intergranular lanes. The figure refers to multi-scale models. The acoustic modes were filtered out prior to analysis. The bins are $100 \mathrm{~km}$ wide

Ploner et al. $(1998,1999)$ and is based on filtering out $p$-modes seen in the $k-\omega$ diagram. We have also carried out the analysis described below without first filtering the $p$-modes. The basic results remain unchanged, but the oscillations lead to a larger scatter, which makes trends more difficult to recognize.

Histograms of relative granule/intergranular-lane counts and their contribution to the total area are plotted in Fig. 30. The major "area" contributors among the granules are those with sizes from 600 to $1300 \mathrm{~km}$ (Fig. 30b).
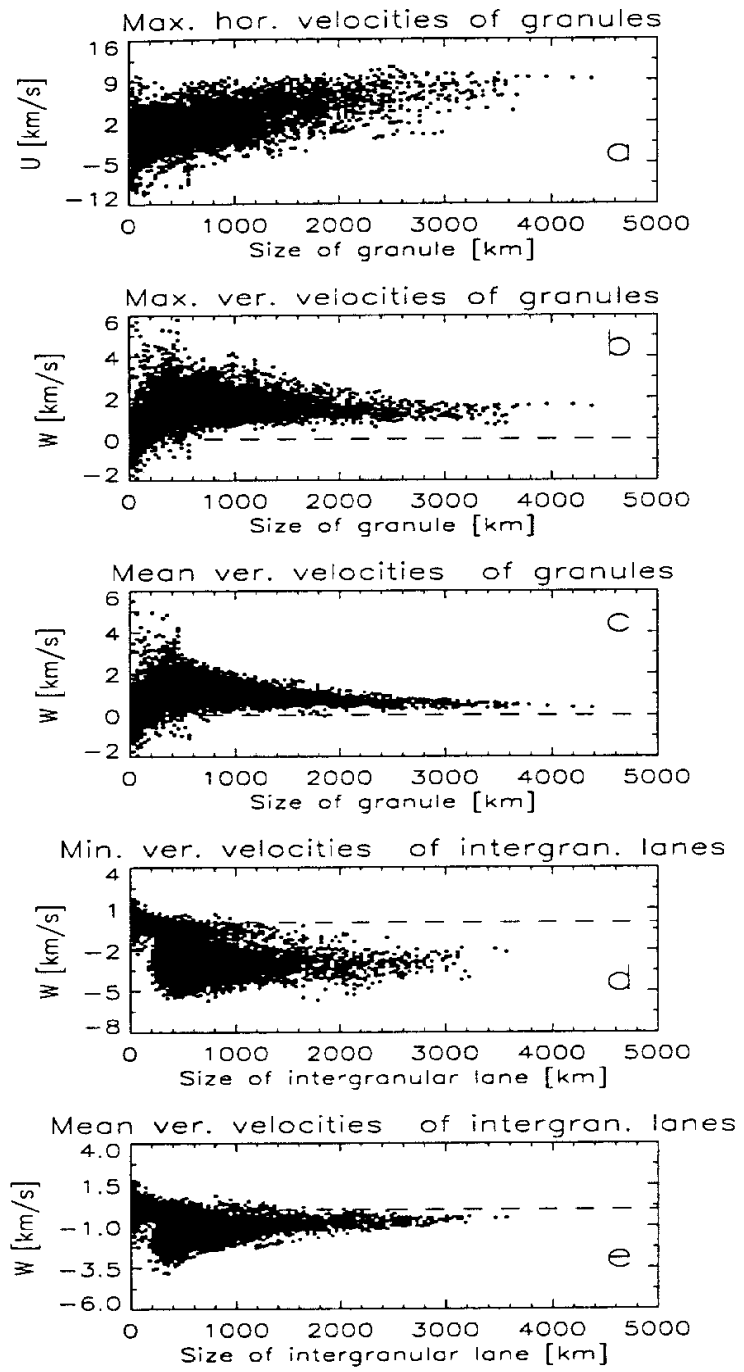

Fig. 31. Maximum horizontal a), maximum b) and mean c) vertical velocities in granules, and minimum d) and average e) vertical velocities in intergranular lanes as a function of their sizes. Plotted are the values at the solar surface $\left(\log \tau_{\mathrm{R}}=0\right)$ in multi-scale models after the removal of p-modes

In the histogram of intergranule counts there is a sharp peak at $500 \mathrm{~km}$. They are also the major contributors to the total "area" of dark lanes (Fig. 30d).

Velocity and temperature properties of granules and intergranular lanes are represented in Figs. 31 and 32, respectively. The striking feature of Fig. 31a is the almost linear dependence of the maximum horizontal velocity on granule size, in general agreement with the mass balance condition (Nelson \& Musman 1978; Nordlund 1985; Steffen et al. 1989), which was also found for ss models (Sect. 3.1.2).

The vertical velocities of granules, shown in Figs. 31b and c, increase with granular size up to $\sim 600 \mathrm{~km}$ and remain unchanged or decrease for larger granules. The small 
granules exhibit a large scatter which can be produced by granules with different evolutionary histories. We do not exclude, however, the influence of model oscillations on behaviour of their velocity field in ms simulations.

Granules are smallest either just after their birth through fragmentation of their parent granule, or shortly before their death through dissolution. The evolution of granules in these ms models has been studied in detail by Ploner et al. (1999) and they find that indeed the mean vertical velocities of small granules near the beginning of their lifetime (stronger upflows) and averaged velocities of the very smallest ones near the ends of their lifetimes (weak upflows) differ significantly. The properties of these very small "granules" in these models are a complicated case for direct study because they are affected by model oscillations significantly and because in non-stationary simulations they additionally depend on prehistory of flow evolution (Ploner et al. 1999).

The vertical velocities of the ss models show qualitatively the same dependence (Fig. 27) on granule respectively lane size, with the exception of the smallest granules. These exhibit a much larger range of velocities, due mainly to the fact that the stable small-scale convection cell exhibit much larger upflows than the small granules formed through fragmentation of a larger granule. Not surprisingly the granules of the highly variable ms simulation exhibit a behaviour more similar to the unstable ss models.

Plotted in Figs. 31d and e are velocities in the intergranular lanes. They suggest the presence of two families of intergranular lanes: those with width above $200 \mathrm{~km}$ and rapid downflows and those which have only weak vertical velocity (which may even correspond to a weak upflow) and which are generally narrower than $200 \mathrm{~km}$. The coexistence of these two families of intergranular lanes is especially clearly seen in Fig. 31d - in the plot of minimum vertical velocities found inside the intergranular lanes. The second family, the narrower, slowly flowing "lanes" are usually associated with the fragmentations of a granule and are the precursors of the new lane formed by the fragmentation (see Ploner et al. 1999). This conclusion is supported by the properties of the lanes formed by the splitting of large ss convective cells. The fact that lanes with strong downward-directed velocities cannot be narrower than roughly $200 \mathrm{~km}$ depends on the mass balance condition and, probably, on the spatial step used, because for ss models with smaller horizontal domain size the well evolved downflows can be practically as narrow as $100 \mathrm{~km}$.

In Fig. 32 the temperature at $\tau_{\mathrm{R}}=1$ of granules and lanes is plotted vs. their size. The granular temperature increases with granular size up to sizes of about $400-600 \mathrm{~km}$ and then remains unchanged (Fig. 32a - for maximal granular temperature) or even decreases on average (Fig. 32b - mean temperature). Note that the scatter in temperature of the small granules is small compared to the scatter exhibited by the velocities in Fig. 31. Temperatures of
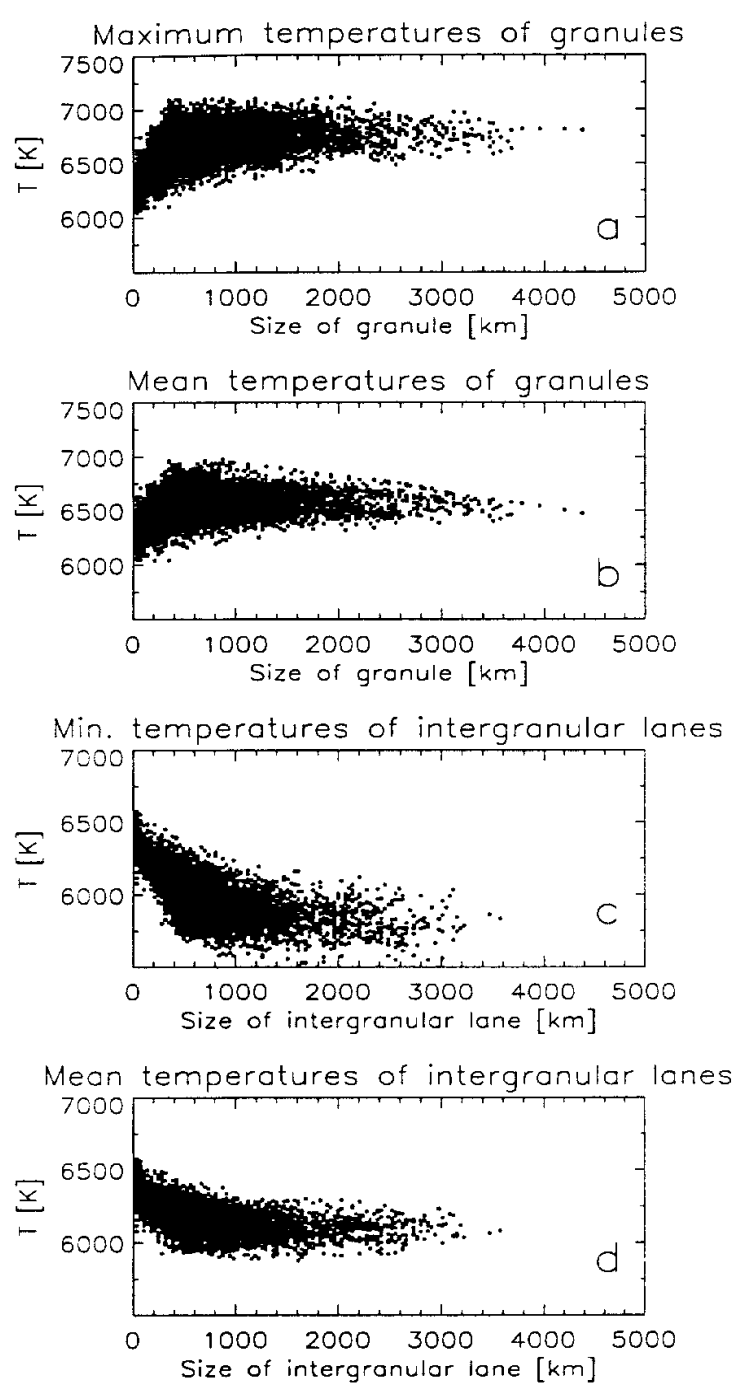

Fig. 32. Size dependence of maximum a) and mean temperatures b) of granules as well as minimum c) and mean d) temperatures of intergranular lanes. Shown are values at the surface, $\log \tau_{\mathrm{R}}=0$, of multi-scale models

intergranular lanes also show two dependences: a sharp decrease with size up to $\sim 700 \mathrm{~km}$ and a slower decrease of their minimum temperatures beyond that (Fig. 32c). Their mean temperatures actually remain unchanged for sizes above $600-800 \mathrm{~km}$.

The scale dependence of temperatures of granules and intergranular lanes, shown in Fig. 32, are similar to those given in Sect. 4.1. for the ss models (Fig. 28). For those models the temperatures of newly formed smallscale granules (intergranular lanes) are close (almost coincident) to those derived from evolved structures.

The fact that many of the smallest granules are cooler than the smallest lanes, although by definition the former are brighter (compare with Fig. 32), is due to the 
different temperature gradient in the two types of features (the gradient is larger in the granules).

The scale size dependence of the intensity, shown in Fig. 33, roughly reflects the same behaviour as seen in Fig. 32 for the temperature. It is nevertheless interesting that the size-independence of the mean intensity of intergranular lanes larger than $600-700 \mathrm{~km}$ (Fig. 33d) is in contrast to the behaviour of the ss models (Fig. 29d).

\subsection{Interpretation of scale-dependent relationships}

In two previous sections we have ascertained that simulated small-scale granules significantly differ in their velocity features if they have different history of the evolution. If small-scale convective flows are well evolved, quasi-stable, and deep-formed then granular velocities, associated with them, decrease with increasing their sizes (circles, Figs. 27a and b). The opposite scale dependence is realized for small-scale granules which become seen at the beginning stage of fragmentation of underlying large-scale thermal upflows (Figs. 27a and b, crosses). In ss modeling they are well separated while in ms modeling the presence of these two sorts of small-scale granules leads to large scatter of granular velocities and velocities of intergranular lanes for region of small-scale structures.

The results described above can be explained by invoking two mechanisms, which act on small- and large-scale structures, respectively.

For small-scale inhomogeneities the smoothing influence of horizontal energy exchange through radiation becomes important (Steffen et al. 1989). This effect is most important near the $\tau=1$ surface. This mechanism causes the temperature difference between small granules and lanes to diminish with decreasing size (see Fig. 32). This also explains the increase of intergranular lane brightness and decrease of granular brightness seen in Fig. 33 with decreasing size.

The second mechanism acts on large-scale upflows. They evolve mainly adiabatically and could in principle have higher temperatures and vertical velocities than is exhibited by the simulations. However, they are effectively retarded by the pressure excess that builds up in granules (Schüssler 1992). This pressure excess causes buoyancy braking, leading to the reduced upflow velocities in larger granules (above a granule size of $1000 \mathrm{~km}$ ) seen in Figs. 31b and c (see also Ploner et al. 1999). In many cases the buoyancy braking concentrates the strongest upflows into certain locations in the large granule, e.g. near its boundary, making the flow (and the brightness structure) inhomogeneous.

Granules are relatively free of both effects only over a relatively narrow range of sizes $(\sim 600-1000 \mathrm{~km}$, these correspond to convection cell sizes of $\sim 900-1500 \mathrm{~km}$ ).
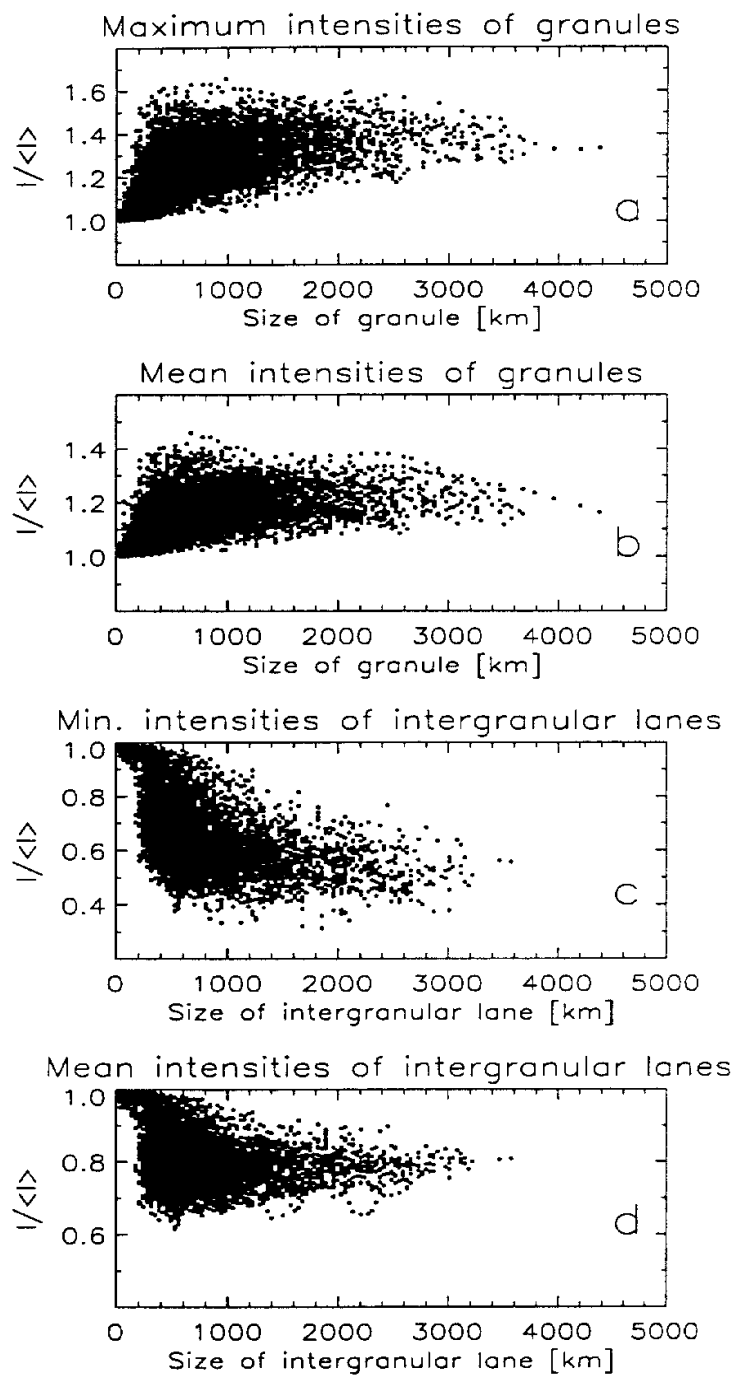

Fig. 33. Dependence of maximal a) and mean b) intensities of granules and minimal c) and mean d) intensities of intergranular lanes on their sizes. The intensity computed at $\lambda 500 \mathrm{~nm}$. Acoustic oscillations were removed from the multi-scale models prior to analysis

\subsection{Comparison with observations}

The scale dependence of the brightness field of our models (Fig. 33) can be compared with the observed distribution of Hirzberger et al. (1997 - their Figs. 8 and 10). Qualitatively theoretical and observational granule data look similar - the granular brightness increases linearly with the size of small-scale granules. It becomes approximately size-independent or even decreasing slightly at larger scales. However, the largest scatter of granular intensities (at the "elbow") is located at smaller scales $(\sim 400-800 \mathrm{~km})$ in our models relative to the observations $(\sim 1500 \mathrm{~km})$. The simulations also predict a linear decrease of brightness of small-scale intergranular lanes, which is hardly visible in the observations, excluding the 

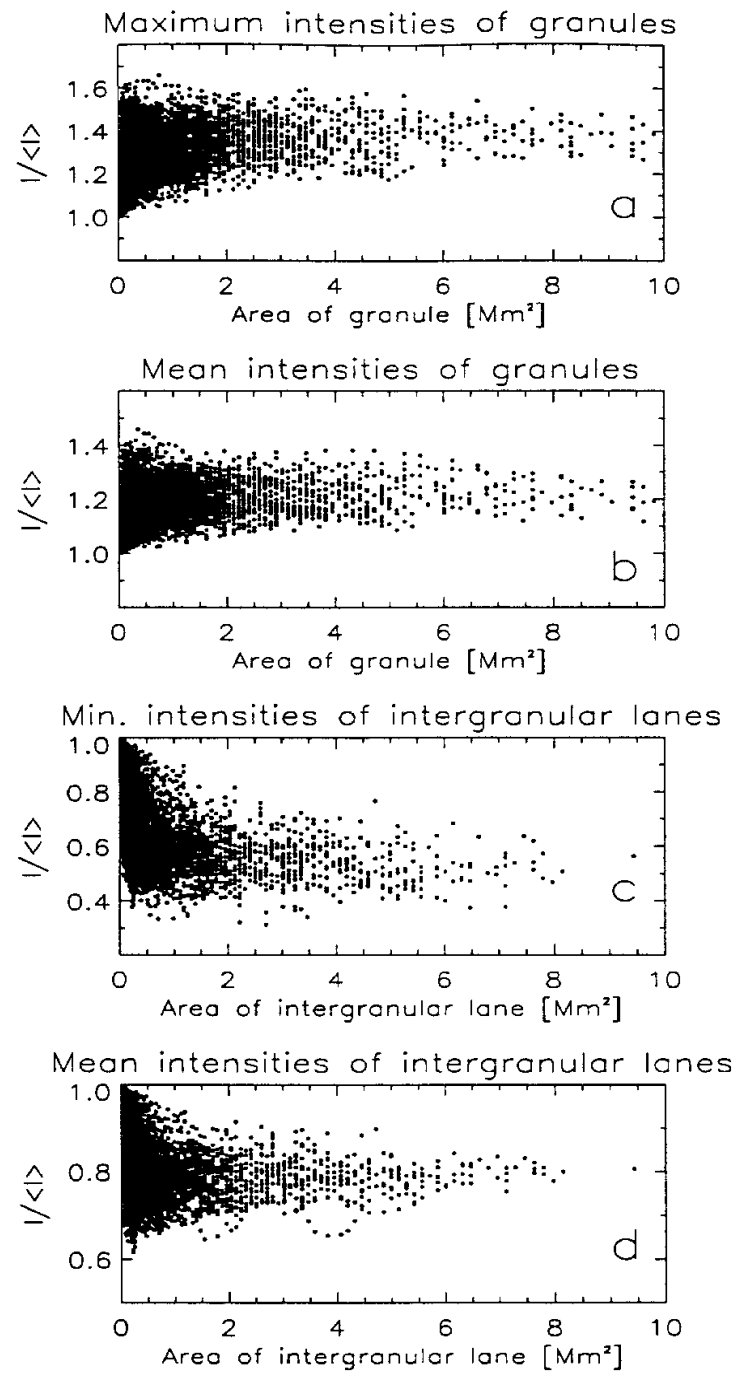

Fig. 34. The data of Figs. 33a and b are replotted as a function of the "area" of granules and intergranular lanes (see text for details)

darkest lanes. Finally, the amplitudes of the intensity fluctuations resulting from the simulations are larger than in the observations.

These differences can be explained by (1) the spatial smoothing of the observed brightness map (due to seeing), (2) different spatial dimensions of the studied intensity fields (2-D in observations and 1-D in the models), and (3) different granule finding algorithms. The high scatter of intensities seen for small granules can be explained in part by the coexistence of flows of different nature located there, i.e. small granules recently formed through fragmentation, or small granules nearing the end of their lifetime. Some of the points may even represent small-scale bright upflows inside larger granules.

To better compare the simulated data with the observations, we have replotted Figs. $33 \mathrm{a}$ and $\mathrm{b}$ in terms of
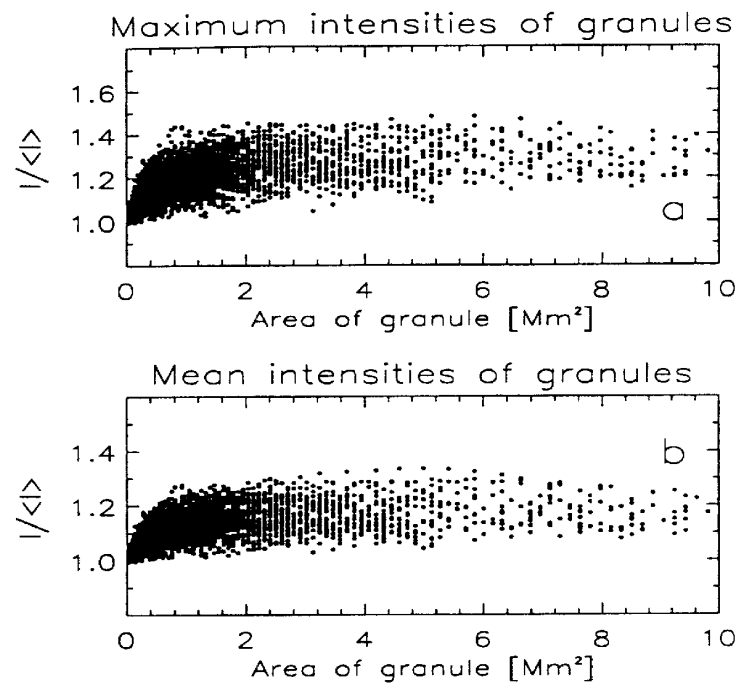

Fig. 35. The data of Fig. 33 are plotted, after spatial smearing to simulate the limited spatial resolution typical of observations (see text), as a function of the "area" of granules

granule area. To find the "area" of 2-D synthetic granules we assumed that they have a circular shape. For intergranular lanes this simple shape presentation can be hardly assumed and is used here mostly in qualitative sense. The new dependencies are shown in Fig. 34.

For granules we can now hardly see any "elbow" at all, only a small decrease of their maximum intensity in the small-scale area range. Narrow intergranular lanes, however, still demonstrate a stronger decrease of brightness towards smaller scales. Therefore, the difference between the present 2-D models and the observational relationships found by Hirzberger et al. (1997) basically results from spatial smoothing and possibly in the differences in the granule finding algorithm.

In Fig. 35 we display the granular data as in Figs. 33 and 34 , but after spatially smearing the emergent intensity. The point spread function (PSF) used consists of the sum of two Gaussians with dispersions of 180 and $540 \mathrm{~km}$, respectively (Deubner \& Mattig 1975). The comparison of this figure with the results of Hirzberger et al. (1997) supports the argument that spatial smearing can explain a significant part of the quantitative disagreement between the simulations and the observations. This implies that the finite spatial resolution of the current observations can in principal distort the true size dependence of granular properties substantially.

The different dimensions of the observed and simulated granular brightness fields probably also play a role. Only the comparison of the results of 3-D simulations with these observations can clarify this point. 


\section{Discussion}

The behaviour of the velocity in the low photosphere of the model is explained by the condition of mass balance which follows from the continuity equation. For simple models of anelastic convective flows in a stratified medium it can be shown (Steffen et al. 1989; Spruit et al. 1990; Schüssler $1992)$ that the horizontal velocity $(U)$ is a function of the horizontal size of the convection cell $(l)$ :

$U \sim W l / H$,

where $W$ is the vertical velocity and $H$ the scale height of the vertical mass flux (vertical momentum density). This proportionality is valid in both the 2-D and 3-D cases. However, in real granular convection $W$ and $H$ are themselves functions of $l$ and the final relation between $U$ and $l$ can be nonlinear. For instance, Steffen et al. (1989) found from their ss models that the maximum rms horizontal velocities could be represented as $U_{\text {rms, max }} \sim \sqrt{L}$, where $L$ is the horizontal size of the model cells. Our simulations, however, give $U_{\text {rms, max }} \sim L^{0.86}$. The differences between their and our results occur for the following reasons.

- Different geometry of the models: Steffen et al. (1989) computed 2-D models in cylindrical geometry, while we employed 2-D Cartesian geometry. In the latter case the horizontal velocities $U$ are larger than in 2-D cylindrical flows or in fully 3-D cartesian geometry. This follows from the continuity equation and is clearly seen in the comparison between our $U_{\text {rms }}$ (Fig. 2a) and those found by Steffen et al. (1989 - their Fig. 2a) or between the present 2-D results and 3-D computations done by Atroshchenko \& Gadun (1994 - their Fig. 1b);

- Differences in the velocity may also result from different upper boundary conditions for velocities. We do not reduce the velocities at the top of the model in any form, while Steffen et al. (1989) postulated that model velocities had to vanish there completely. We expect that this affects the sensitivity of model properties to the convection cell sizes.

In all the 2-D models of Steffen et al. (1989) $U_{\text {rms }}$ reaches its maximum approximately at the same height in the photosphere. At this height the vertical velocity is roughly scale-independent.

In our model atmospheres we find that $H \sim L^{0.14}$. The vertical velocities are different at these heights. For instance, below the surface $W_{\mathrm{rms} \text {,max }}$, shown in Fig. $3 \mathrm{~b}$, can be approximated by $W_{\text {rms,max }} \sim L^{-0.13} ; W_{\text {rms }}$ in the lower photosphere (at a fixed geometrical height of about $50 \mathrm{~km}$ above the surface) is scale-independent: $W_{\mathrm{rms}} \sim L^{-0.02}$; while in the middle photosphere $(\sim 150-200 \mathrm{~km}) W_{\mathrm{rms}}$ is roughly $L^{0.25}$. If we assume scale-independent $W_{\text {rms }}$ then from (6) we get $U_{\mathrm{rms}} \sim L^{0.86}$, in good agreement with the relation for $U_{\text {rms,max }}$ that we found directly from the simulations. Note, however, that the maxima of $U_{\text {rms }}$ occurs in our models at different photospheric heights for different cell size (Fig. 4a).
Consider now the density inversion exhibited by at least our ss models (Fig. 5c). A surface density inversion is also found in models of stellar convective envelopes based on mixing length theory (Böhm-Vitense 1958). For instance, for stars with masses smaller than $4 M_{\odot}$ Ergma (1970) obtained that their density inversion was most pronounced when the effectiveness of convection was low. Under effectiveness of convection we understand here the ratio of energy transported by a convective element to the energy lost due to radiative exchange.

In the present 2-D simulations the ratio of transported energy to radiative losses depends on the size of the convection cell. The smallest convection cells represent convective transport of energy with a large loss of energy through radiation. According to Fig. $5 \mathrm{c}$ these are also the models with the largest density inversion. Thus 2-D simulations and predictions of mixing-length theory are in good agreement with each other in this sense.

The occurrence of a density inversion when the effectiveness of convection is low can be explained as follows: ineffective convection means a larger role of radiation in energy transfer, producing steeper temperature gradients. Since, on average, the pressure must always grow with depth to compensate for the gravity force, at the peak of the temperature gradient the density is expected to show an inversion according to the equation of state.

\section{Conclusion}

We have analyzed time-dependent sets of 2-D HD models to obtain size-dependent properties of simulated thermal convection and granulation under solar conditions. The two sets of models treat thermal convection as steadystate convection rolls or as highly dynamic, non-stationary convection. Our basic results can be summarized as follows.

Single-scale convection cells can be divided into 3 groups, depending on their sizes: small-scale (up to $\sim 900 \mathrm{~km})$, intermediate-scale $(1000-1500 \mathrm{~km}$, these correspond to sizes of granules in the range of $\sim 600-1000 \mathrm{~km}$ ) and large-scale (larger than $1500 \mathrm{~km}$ ) convection cells. Thermal damping due to radiative transfer in horizontal direction determines the properties of the first group to a large extent: their temperature and brightness fluctuations decrease very rapidly with decreasing size. Upflows in the large-scale convection cells are retarded by pressure excess (buoyancy braking), which determines their evolution: large computed granules end basically by fragmentation. In a narrow size range between these two extremes are cells inside which both these effects have a minimum impact on the upflows. This can be deduced from the dependence of granular properties on their size.

We have studied in detail the importance of the zone of high convective instability associated with the partial 
ionization of hydrogen: ionization effects increase the convective instability of thermal plumes and protect them from thermal damping. There is an asymmetry between hot rising and cool sinking flow in the instability and thermal damping mechanisms (in upflows the ionization effects increase the instability of upflows before at the solar surface radiative damping reduces it again. In downflows the opposite sequence of process takes place). As a consequence for upflows the work of buoyancy force in this zone is roughly independent of their horizontal size, while cool downflows, influenced by radiative heating, show a very strong dependence on their size.

Both kinds of models show a density inversion in subphotospheric layers. It is more pronounced in small convection cells and inside upflows.

From an observational point of view our investigation demonstrate that we can distinguish between nonstationary multi-scale convection and quasi-steady convection from the size dependence of the vertical velocities of small granules at the solar surface. However, they cannot be distinguished in the dependence of temperature or emergent intensity of granules or lanes.

We have compared brightness properties of 2-D simulated granulation with real observations of Hirzberger et al. (1997) and found them to be in qualitative agreement as far as granules are concerned.

For intergranular lanes the theoretical prediction that the brightness of small-scale inhomogeneities decreases linearly with increasing size finds observational confirmation only for the darkest lanes. We expect that the quantitative disagreement between simulations and observations for the range of small-scale lanes may result, at least partially, from problems faced by the observations due to spatial smoothing (produced by seeing and finite telescope resolution) and possible shortcomings from the granule finding algorithm. Simple tests confirm the significant influence of spatial smearing. Hence, based on the simulations presented here we conclude that determining the true size dependence of granular brightness field is beyond the spatial resolution of current observations.

On the other hand, the different dimensions of the studied theoretical (1-D) and observed (2-D) intensity fields are almost certainly responsible for a part of the discrepancy.

Acknowledgements. A. Gadun and S.R.O. Ploner are grateful to the Swiss National Science Foundation for supporting this work. A. Hanslmeier, K. Puschmann, A. Gadun, and K. Pikalov thank the Austrian and the Ukrainian Academies of Sciences for financing the exchange of scientists. A. Hanslmeier acknowledges the financial support from the Austrian Fonds zur Förderung der wissenschaftlichen Forschung. The authors thank Prof. A. Getling for useful comments.

\section{References}

Altrock R.C., 1976, Solar Phys. 47, 517

Asplund M., 2000, A\&A (submitted)

Asplund M., Nordlund Å., Trampedach R., Stein R.F., 2000a, A\&A (submitted)

Asplund M., Nordlund Å., Trampedach R., Allende Prieto C., Stein R.F., 2000b, A\&A (submitted)

Atroshchenko I.N., Gadun A.S., 1994, A\&A 291, 635

Banos G., Nesis A., 1990, 232, 231

Belotserkovskiy O.M., Davydov Yu.M., 1982, Large-Particle Method in Gasdynamics, Nauka, Moscow

Böhm-Vitense E., 1958, Z. Astrophys 46, 108

Cattaneo F., Hurlburt N.E., Toomre J., 1989, in: Solar and Stellar Granulation, Rutten R.J. and Severino G. (eds.). Kluwer, Dordrecht, p. 415

Cattaneo F., Hurlburt N.E., Toomre J., 1990, ApJ 349, L63

Cattaneo F., Brummell N.H., Toomre J., Malagoli A., Hurlburt N.E., 1991, ApJ 370, 282

Deubner F.L., Mattig W., 1975, A\&A 45, 167

Dravins D., Larsson B., Nordlund A., 1986, A\&A 158, 83

Dravins D., Lindegren L., Nordlund Å., 1981, A\&A 96, 345

Ergma E., 1970, Scientific information of AS of USSR 16, 32

Gadun A.S., 1986, Simulation of Turbulent Convection in the Solar Envelope, Inst. Theor. Phys., Kiev, preprint No. 86$106 \mathrm{P}$

Gadun A.S., 1995, Kinemat. i Fiz. Nebesn. Tel 11, No. 3, 54

Gadun A.S., Hanslmeier A., Pikalov K.N., 1997, A\&A 320, 1001

Gadun A.S., Pavlenko Ya.V., 1997, A\&A 324, 281

Gadun A.S., Solanki S.K., Johannesson A., 1999, A\&A 350, 1018

Gadun A.S., Vorob'yov Yu.Yu., 1996, Astronomy Reports 40, 569 (also 1995, Solar Phys. 159, 45)

Grossmann-Doerth U., Schüssler M., Steiner O., 1998, A\&A 337,928

Hanslmeier A., Mattig W., Nesis A., 1991, A\&A 251, 669

Hart M.H., 1973, ApJ 184, 587

Hirzberger J., Vázquez M., Bonet J.A., Hanslmeier A., Sobotka M., 1997, ApJ 480, 406

Hurlburt N.E., Toomre J., Massaguer J.M., 1984, ApJ 282, 557

Hurlburt N.E., Toomre J., Massaguer J.M., 1986, ApJ 311, 563

Karpinskiy V.N., Pravdjuk L.M., 1998, Kinemat. i Fiz. Nebesn. Tel 14, No. 2, 119

Kawaguchi I., 1980, Solar Phys. 65, 207

Keil S.L., Canfield R.C., 1978, A\&A 70, 169

Kneer F.J., Mattig W., Nesis A., Werner W., 1980, Solar Phys. 68,31

Kurucz R.L., 1979, ApJS 40, 1

Kurucz R.L., 1993, Opacities for Stellar Atmospheres: [+0.0], $[+0.5],[+1.0]$. CD-ROM 2

Malagoli A., Cattaneo F., Brummell N.H., 1990, ApJ 361, L33

Massaguer J.M., Zahn J.P., 1980, A\&A 87, 315

Nelson G.D., Musman S., 1977, ApJ 214, 912

Nelson G.D., Musman S., 1978, ApJ 222, L69

Nesis A., Bogdan T.J., Cattaneo F., Hanslmeier A., Knölker M., Malagoli A., 1992, ApJ 399, L99

Nesis A., Hammer R., Kiefer M., Schleicher H., Sigwarth M., Staiger J., Å., 1999, 345265 
Nordlund Å., 1984, in: Small-Scale Dynamical Processes in Quiet Stellar Atmospheres, Keil S.L. (ed.), SPO, Sunspot, p. 174

Nordlund A., 1985, in: Theoretical Problems in High Resolution Solar Physics, Schmidt H.U. (ed.), MPI, München, p. 1

Ploner S.R.O., Solanki S.K., Gadun A.S., Hanslmeier A., 1998, Space Sci Rev. 85, 261

Ploner S.R.O., Solanki S.K., Gadun A.S., 1999, A\&A 352, 679

Rast M.P., 1995, ApJ 443, 863

Rast M.P., 1998, J. Fluid Mech. 369, 125

Rast M.P., Nordlund Å., Stein R.F., Toomre J., 1993, ApJ 408, L53

Rast M.P., Toomre J., 1993a, ApJ 419, 224

Rast M.P., Toomre J., 1993b, ApJ 419, 240

Schüssler M., 1992, in: The Sun — a Laboratory for Astrophysics, Schmelz J.T., Brown J.C. (eds.). Kluwer, Dordrecht, p. 81
Solanki S.K., Rüedi I., Bianda M., Steffen M., 1996, A\&A 308, 623

Spruit H.C., Nordlund Å., Title A.M., 1990, ARA\&A 28, 263

Steffen M., 1989, Spectroscopic Properties of Solar Granulation Obtained from 2-D Numerical Simulations. In: Solar and Stellar Granulation, Rutten R.J. and Severino G. (eds.). Kluwer, Dordrecht, p. 425

Steffen M., 1991, in: Stellar Atmospheres: Beyond Classical Models, Crivellari L., Hubeny I., Hummer D.G. (eds.). Kluwer, Dordrecht, p. 247

Steffen M., Freytag B., 1991, Rev. Mod. Astron. 4, 43

Steffen M., Ludwig H.-G., Krüß A., 1989, A\&A 213, 371

Stein R.F., Nordlund Å., 1998, ApJ 499, 914

Stein R.F., Nordlund Å., Kuhn J.R., 1989, in: Solar and Stellar Granulation, Rutten R.J., Severino G. (eds.). Kluwer, Dordrecht, p. 381

Wöhl H., Nordlund Å., 1985, Solar Phys. 97, 213 